\title{
Répteis da Estação Ecológica Serra Geral do Tocantins, Brasil Central
}

\author{
Renato Sousa Recoder ${ }^{1,5}$, Mauro Teixeira Junior ${ }^{1}$, Agustín Camacho', Pedro Murilo Sales Nunes ${ }^{\text {, }}$
}

Tamí Mott', Paula Hanna Valdujo ${ }^{3}$, José Mario Ghellere ${ }^{1}$, Cristiano Nogueira ${ }^{4}$ \& Miguel Trefaut Rodrigues ${ }^{1}$

${ }^{1}$ Departamento de Zoologia, Instituto de Biociências, Universidade de São Paulo - USP, Rua do Matão, Trav. 14, $n^{\circ}$ 321, Cidade Universitária, CP 11461, CEP 05508-090, São Paulo, SP, Brasil

${ }^{2}$ Universidade Federal de Mato Grosso - UFMT, Av. Fernando Correa da Costa, s/n, Coxipó, CEP 78060-900, Cuiabá, MT, Brasil

${ }^{3}$ Departamento de Ecologia, Instituto de Biociências, Universidade de São Paulo - USP,

Rua do Matão, Trav. 14, $n^{\circ}$ 321, Cidade Universitária, CEP 05508-090, São Paulo, SP, Brasil

${ }^{4}$ Conservação Internacional do Brasil, Programa Cerrado-Pantanal, Departamento de Zoologia, Universidade de Brasília - UnB, CEP 70910-900, Brasília, DF, Brasil

${ }^{5}$ Autor para correspondência: Renato Sousa Recoder,e-mail: renatorecoder@gmail.com.

RECODER, R.S.,TEIXEIRA JUNIOR, M., CAMACHO, A., NUNES, P.M.S., MOTT, T., VALDUJO, P.H., GHELlERE, J.M., NOGUEIRA, C. \& RODRIGUES, M.T. Reptiles of Serra Geral do Tocantins Ecological Station, Central Brazil. Biota Neotrop. 11(1): http://www.biotaneotropica.org.br/v11n1/en/ abstract?article+bn03611012011.

\begin{abstract}
The South American Cerrado harbors a rich reptilian fauna with high number of endemics. However, knowledge of this diversity is still incipient in front of accelerated transformation of natural landscapes, which makes the domain a "hotspot" for study and conservation of global biodiversity. Studies on the reptile fauna of Cerrado are urgently needed for a better understanding of the processes that led to their origin and distribution and to support conservation planning. We used standard methods to sample two unexplored regions of the Serra Geral do Tocantins Ecological Station (EESGT), located in the Jalapão region of the Cerrado domain. We recorded 45 species of reptiles for the EESGT and surroundings, which represents high species richness, comparable to other well-sampled regions of the Cerrado. Accumulation curves and estimators indicate that sampled richness of lizards and amphisbaenas are close to real values. However, current sampling underestimates snake richness. The non-random distribution of species in the landscape agrees with previous evidence suggesting differential use of habitats by Cerrado reptiles. Combining the results of this study with those of previous surveys conducted in the region, we recorded 88 species of reptiles for the Jalapão region with eight new records, including Bachia oxyrhina, a recent described species of this region. We found three general patterns of distribution: (1) species endemic of the Cerrado domain, (2) species shared with the domains of the South American diagonal of tropical open formations, (3) species widely distributed, also shared with forest ecosystems. Species of wide distribution prevails in the region, but there is large number of species typical of the Cerrado, possibly including five endemics to Jalapão, and there is an important contribution of the Caatinga fauna. The distribution of reptile species in local and regional scales shows the need to consider the landscape heterogeneity for adequate conservation planning in the Cerrado region. Because of the physical and biotic characteristics, EESGT has a key role in the preservation and knowledge of the diversity of Cerrado reptiles.
\end{abstract}

Keywords: Cerrado, Squamata, lizards, snakes, amphisbaenas, diversity, Jalapão, conservation.

RECODER, R.S.,TEIXEIRA JUNIOR, M., CAMACHO, A., NUNES, P.M.S., MOTT, T., VALDUJO, P.H., GHELlere, J.M., NOGUEIRA, C. \& RODRIGUES, M.T. Répteis da Estação Ecológica Serra Geral do Tocantins, Brasil Central. Biota Neotrop. 11(1): http://www.biotaneotropica.org.br/v11n1/pt/ abstract?article+bn03611012011.

Resumo: Os Cerrados sul-americanos abrigam alta diversidade de répteis, incluindo elevado número de endemismos. No entanto, o conhecimento desta diversidade é ainda incompleto frente à acelerada transformação das paisagens naturais no Brasil central. Constituem, portanto, uma das regiões prioritárias para estudo e conservação da biodiversidade mundial. Estudos intensivos sobre a fauna de répteis do Cerrado são necessários e urgentes para melhor compreensão dos processos que levaram à sua origem e distribuição e para subsidiar ações de conservação. Por meio de métodos padronizados, amostramos duas regiões ainda inexploradas da Estação Ecológica Serra Geral do Tocantins, situada na região do Jalapão. Registramos 45 espécies de répteis para a EESGT e entorno, o que representa uma riqueza alta e comparável à de outras regiões bem amostradas do Cerrado. Curvas de acumulação e estimadores indicam que a riqueza local de lagartos e anfisbenídeos aproxima-se da riqueza real enquanto a de serpentes é subestimada. A distribuição não-aleatória das espécies na paisagem concorda com evidências anteriores sugerindo utilização diferencial dos hábitats pelos répteis. Reunindo os resultados do presente estudo com os de levantamentos prévios realizados na região, registramos 88 espécies de répteis para o Jalapão sendo oito registros novos que incluem Bachia oxyrhina uma espécie recém descrita da região. As espécies da área 
apresentam três padrões gerais de distribuição: (1) espécies endêmicas do Cerrado, (2) espécies compartilhadas com domínios da diagonal de formações abertas sul-americanas, e (3) espécies de ampla ocorrência, compartilhadas também com ecossistemas florestais. Prevalecem espécies de ampla distribuição, porém é grande o número de espécies típicas do Cerrado, incluindo cinco possivelmente endêmicas do Jalapão, e há contribuição importante da fauna da Caatinga. A distribuição dos répteis em escala local e regional demonstra a necessidade de considerar a heterogeneidade paisagística para o planejamento de diretrizes visando à conservação em regiões do Cerrado. Por sua grande extensão, posição biogeográfica e complexidade de relevo e tipos de hábitat, a EESGT tem papel fundamental para a preservação e conhecimento da diversidade de répteis do Cerrado.

Palavras-Chave: Cerrado, Squamata, lagartos, serpentes, anfisbenas, diversidade, Jalapão, conservação.

\section{Introdução}

Historicamente o Cerrado tem sido pouco reconhecido em termos de potencial faunístico quando comparado aos ambientes florestais neotropicais (Mares 1992). Antigamente a fauna do Cerrado era considerada sem características próprias (Sick 1965) e a diversidade de répteis depauperada (Vitt 1991) composta essencialmente por espécies generalistas e de distribuição ampla ao longo da diagonal de formações abertas da América do Sul (Vanzolini 1963, 1976, 1988). Esta visão simplista da fauna do Cerrado foi em parte responsável pela falta de interesse em realizar amostragens intensivas em sua área, o que levou, por muitas décadas, a uma falta de valorização de sua biodiversidade em estratégias de conservação (Redford et al. 1990).

Contudo, com o aumento no número de inventários, revisões taxonômicas e o emprego de novos métodos de amostragem tem-se demonstrado que os répteis, tradicionalmente incluindo crocodilianos, quelônios, serpentes, anfisbenídeos e lagartos, representam grupo diverso e característico do domínio dos Cerrados sul-americanos. Além disso, esse grupo apresenta elevada riqueza de espécies (Colli et al. 2002, Nogueria et al. 2010), especificidade no uso de hábitats (Nogueira et al. 2009) e inúmeros casos de endemismos (Rodrigues 1987, Colli et al. 2002, Nogueira et al. 2010).

O Cerrado é o segundo maior domínio morfoclimático da América do Sul e maior savana neotropical (Ab’Sáber 1977). Estendia-se originalmente por cerca de dois milhões de $\mathrm{km}^{2} \mathrm{e}$ ocupa uma posição central no continente, abrangendo $22 \%$ do território brasileiro (Oliveira \& Marquis 2002). Suas paisagens são caracterizadas pela presença de tipos variáveis de hábitats, distribuídos em mosaico e formando um complexo heterogêneo de formações vegetais campestres, savânicas e florestais (Eiten 1972, Oliveira-Filho \& Ratter 2002). O clima é altamente sazonal, com estações secas e chuvosas bem demarcadas e baixa amplitude térmica anual (Nimer 1989).

Pelas características físicas, heterogeneidade ambiental e alta diversidade de espécies, o Cerrado representa uma região distinta e de enorme importância biológica nos trópicos (Oliveira \& Marquis 2002, Scariot et al. 2005). No entanto, seus hábitats naturais têm sido intensamente transformados por ação antrópica e grande parte da cobertura vegetal nativa foi substituída, principalmente por pastagens e monoculturas extensivas (Ratter et al. 1997, Klink \& Moreira 2002). As estimativas mais recentes sobre a cobertura original de área do Cerrado apontam para perdas substanciais (Myers et al. 2000, Cavalcanti \& Joly 2002, Klink \& Machado 2005, Mittermeier et al. 2005, Silva et al. 2006) e sugerem a existência de apenas cerca de $30 \%$ da cobertura de vegetação original. Ademais, somente 5,5\% do domínio estariam protegidos em unidades de conservação, com menos de 3\% em áreas de proteção integral (Mittermeier et al. 2005).

Por abrigar elevada diversidade biológica em área altamente ameaçada, o Cerrado é considerado um dos hotspots para o estudo e conservação da biodiversidade mundial (Myers et al. 2000). No entanto ainda existe escassez de informações biológicas robustas sobre a composição das comunidades de répteis para extensas áreas do domínio. Assim, estudos intensivos sobre a fauna de répteis do Cerrado são necessários e urgentes para maior compreensão dos processos ecológicos e evolutivos que levaram à sua origem e distribuição e subsidiar ações de conservação (Colli et al. 2002, Rodrigues 2005a,b, Nogueira et al. 2010).

Neste estudo, apresentamos os resultados de um levantamento intensivo da fauna de répteis realizado na Estação Ecológica Serra Geral do Tocantins, unidade de conservação inserida no Jalapão, região de grande importância e ainda pouco perturbada no Cerrado (Proença et al. 2002). Este estudo complementa informações anteriores obtidas na área (e.g., Vitt et al. 2005, Mesquita et al. 2006, Nogueira 2006, Vitt et al. 2007) e preenche importante lacuna de informação, apresentando dados robustos sobre a riqueza, organização e relações faunísticas da comunidade de répteis da região.

\section{Material e Métodos}

\section{1. Área de estudo}

A região do Jalapão localiza-se na porção leste do Estado do Tocantins (TO) estendendo-se pelas porções sul do Estado do Maranhão (MA) e Piauí (PI) e oeste do Estado da Bahia (BA), em uma zona do domínio do Cerrado em tensão ecológica com o domínio das Caatingas (Arruda \& von Behr 2002). Abrange $53.300 \mathrm{~km}^{2} \mathrm{de}$ cerrados bem conservados e concentra grandes áreas contínuas de reservas estaduais e federais, formando o maior conjunto de áreas protegidas no Cerrado brasileiro (Cavalcanti \& Joly 2002). A região caracteriza-se pela presença de morros-testemunho e extensas depressões preenchidas por areias quartzosas originadas do intemperismo fluvio-eólico dos planaltos areníticos da Serra Geral e Chapada das Mangabeiras, que servem como divisor de águas entre as bacias dos Rios Tocantins e São Francisco (Faleiro 2002, Tocantins 2003, Rodrigues et al. 2008, Ribeiro et al. 2009).

A Estação Ecológica Serra Geral do Tocantins (EESGT) preserva uma área de 716 mil ha na região do Jalapão entre os municípios de Almas, Ponte Alta e Mateiros no Tocantins e Formosa do Rio Preto na Bahia (Figura 1). Existem na estação dois níveis de superfícies altimétricas: um inferior, na porção central e ao sul da estação com altitudes entre 400 e $600 \mathrm{~m}$, caracterizado por amplas depressões formadas por áreas de deposição delimitadas por furnas, entre as quais despontam morros relictuais isolados; e outro nível superior, de planaltos e chapadas com altitudes entre 700 e $900 \mathrm{~m}$ ao leste da estação e pertencente à Serra Geral (Rodrigues et al. 2008, Ribeiro et al. 2009) (Figura 2). Este relevo escalonar é resultado da erosão diferencial dos arenitos mesozóicos da formação Urucuia, que formam o embasamento rochoso das serras da região, resultando em depressões cobertas por neossolos-quartizarênicos provenientes da erosão da chapada e solos argilo-arenosos mais profundos por sobre os planaltos (SEPLAN 2003). As paisagens apresentam as fisionomias típicas do Cerrado (Oliveira-Filho \& Ratter 2002) com predominância de campos e cerrados abertos sobre solos arenosos, porém também 


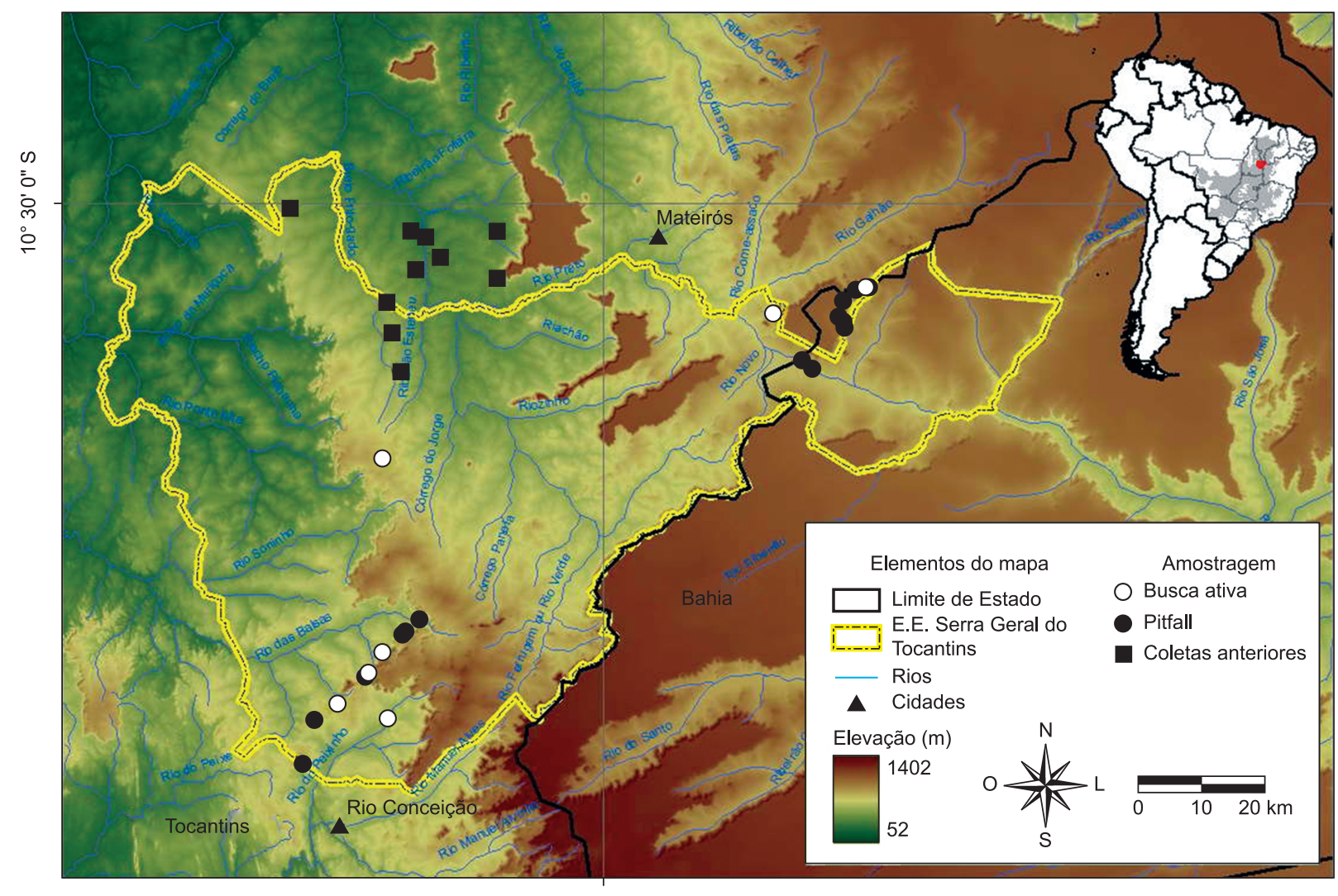

$46^{\circ} 30^{\prime} 0^{\prime} \mathrm{W}$

Figura 1. Mapa da região sul do Jalapão, divisa do Tocantins com a Bahia. Linha amarela demarca os limites da EESGT (Estação Ecológica Serra Geral do Tocantins). Os círculos pretos indicam os pontos de instalação das linhas de armadilhas de interceptação-e-queda, os círculos brancos indicam os pontos amostrados por busca ativa e os quadrados pretos as coletas anteriores realizadas na região.

Figure 1. Map of southern portion of Jalapão region, border of Tocantins and Bahia. Yellow line marks the limits of EESGT (Serra Geral do Tocantins Ecological Station). Black circles indicate points of installation of the pitfall traps, white circles indicate the points of active search, and black squares indicate points of previous collections.

podem sem encontrados irregularmente distribuídos cerrados mais densos, cerrados sobre áreas rochosas de deposição coluvionar nas vertentes dos morros, e veredas e matas de galerias associadas a rios.

Duas regiões distintas da EESGT foram amostradas (Figura 1): porção sul, pertencente ao município de Almas (TO), localizada nas depressões da estação, amostrada entre 27 de janeiro e 4 de fevereiro de 2008; e porção leste, pertencente ao município de Formosa do Rio Preto (BA), situada nos planaltos da Serra Geral da Bahia, amostrada entre 8 e 16 de fevereiro de 2008.

\section{Métodos de amostragem}

A fauna de répteis foi amostrada com conjuntos de armadilhas de interceptação-e-queda com cercas-guia, complementada por coletas ativas. Cada conjunto de armadilhas de queda (pitfall traps) consistiu de quatro baldes de $35 \mathrm{~L}$, dispostos radialmente, enterrados ao nível do solo e unidos por cercas verticais de lona plástica de cerca de $4 \mathrm{~m}$ (Figuras 3 e 4). Em cada área estudada da estação foram utilizados nove pontos amostrais compostos por linhas de armadilhas, cada uma delas contendo cinco conjuntos (20 baldes). As coletas ativas incluíram a busca de indivíduos abrigados em microhábitats específicos como rochas, troncos caídos, cupinzeiros e sob serapilheira e a busca visual de indivíduos ativos. Deste modo, a amostragem diária consistiu na coleta realizada por 180 baldes complementada por buscas intensivas por exemplares ativos (não quantificado), totalizando um esforço de 1560 baldes/dia na porção sul da EESGT e 1620 baldes/dia na porção leste (Tabela 1). A amostragem foi complementada com o encontro ocasional de espécimes nos dias próximos aos períodos de amostragem e com a ajuda de exemplares trazidos por terceiros. A amostragem de serpentes foi favorecida pelo método de busca de exemplares ao longo de estradas (Fitch 1949). Os exemplares capturados foram mortos com injeção de lidocaína, preservados em formol 10\%, transferidos para álcool $70 \%$ e incorporados à coleção herpetológica do Museu de Zoologia da Universidade de São Paulo, São Paulo (MZUSP). Amostras de fígado e/ou músculo foram obtidas de cada espécime coletado e depositadas no Banco de Tecidos do Instituto de Biociências da Universidade de São Paulo, São Paulo.

\section{Hábitats amostrados}

Na região sul da estação os nove pontos amostrais foram distribuídos de forma a contemplar os seguintes hábitats (Tabela 1, Figura 4): campos de gramíneas sobre solo arenoso, com quantidade variável de serapilheira, presença de arbustos, e muitos espaços de solo exposto (linhas 1, 2 e 5); mata de galeria, com dossel bem definido, estrato arbustivo ou herbáceo pouco desenvolvido, ao longo de pequeno riacho (linha 3); campo úmido com grande densidade de gramíneas, pouco solo exposto e poucos arbustos, adjacente a vereda (linha 4); cerrado típico aberto, sob influência de encosta de morro, com presença esparsa de árvores, rochas, arbustos, grande quantidade de solo arenoso exposto e pequena camada de serapilheira (linhas 6 e 8); e cerrado típico denso, com grande quantidade de 

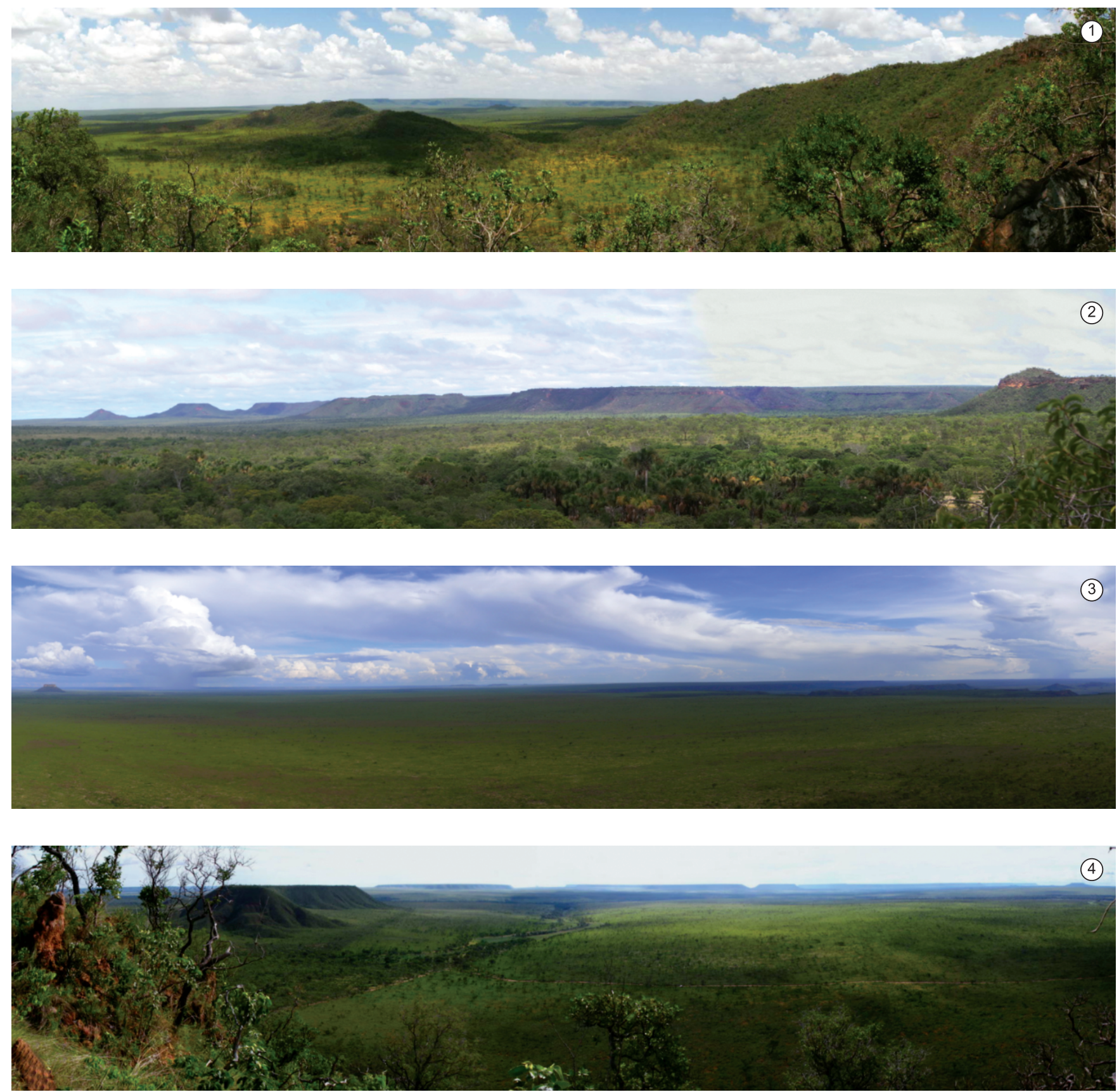

Figura 2. Paisagens na EESGT: 1. Morros residuais e cerrados típicos e pedregosos, na porção sul da EESGT; 2. Chapadões isolados, matas e cerrados densos, na porção sul da estação; 3. Vista da porção central da EESGT tomada do alto do Morro do Fumo, mostrando amplas áreas de deposição de areia com campos, morro testemunho isolado (esquerda) e furnas (direita); 4. Morros testemunho, chapadões e áreas de deposição entrecortadas por drenagens, na porção leste da estação. (Fotos: 1, 4 - M. Teixeira Jr.; 2 - M.T. Rodrigues; 3 - R.S. Recoder).

Figure 2. Landscapes in EESGT: 1. Relictual hills and typical and rocky savanna habitats, in the southern portion of EESGT; 2. Isolated plateaus, dense forests and savannas, in the southern portion of the station; 3. View of the central portion of the EESGT taken from the top of "Morro do Fumo" hill, showing large areas of sand deposition with grasslands, relictual hills (left) and depressions (right); 4. Relictual hills, high plateaus and depositional areas interspersed with drainages, in the eastern portion of the station. (Photos: 1, 4 - M. Teixeira Jr., 2 - M.T. Rodrigues, 3 - R.S. Recoder).

árvores e serapilheira, poucos arbustos e gramíneas, solo arenoso, e com presença de troncos caídos (linhas 7 e 9).

Na região leste da estação foram amostrados os seguintes hábitats: campos com solo argilo-arenoso, com quantidade variável de arbustos e serapilheira, árvores esparsas, densidade variável de gramíneas com grande quantidade de solo exposto (linhas 1, 2, 4 e 5); cerrado típico com grande quantidade de serapilheira, presença de árvores formando cobertura, pouco solo exposto e grande quantidade de troncos caídos e cupinzeiros (linha 3); cerrado pedregoso em contato com encosta de morro, com grande quantidade de conglomerados areníticos, gramíneas e pouca presença de serapilheira, troncos caídos, arbustos, e árvores esparsas (linha 6); cerrado típico com influência de encosta, 


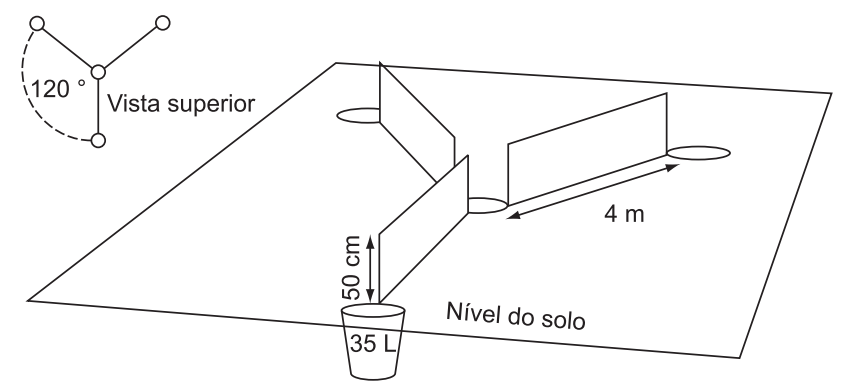

Figura 3. Esquema de montagem de armadilhas de interceptação-e-queda (pitfall traps). Cada estação é composta de quatro baldes dispostos radialmente ao nível do solo e unidos por cercas-guia de lona, amparadas por estacas de madeira.

Figure 3. Arrangement of the pitfall traps. Each sampling unit is composed of four buckets in radial arrangement at ground level and connected by plastic drift-fences and supported by wood stakes.

com muitas rochas, grande densidade de gramíneas, e presença de árvores esparsas e arbustos (linha 7); e campos arenosos adjacentes a campos úmidos e veredas, com variável proporção de gramíneas e solo exposto, com poucos arbustos e muitos cupinzeiros (linhas 8 e 9).

\section{Análises}

Para avaliar o esforço de coleta despendido foram elaboradas curvas de rarefação de espécies baseadas em 10.000 randomizações de uma matriz contendo espécies amostradas nas linhas e os dias de amostragem nas colunas, com o programa EstimateS v.8.0.0 (Colwell 2006). As curvas não foram re-escalonadas para indivíduos, como recomendado por Gotelli \& Colwell (2001), de modo a poder comparar os esforços de amostragem de lagartos e serpentes, contornando assim a maior dificuldade relativa envolvida na amostragem e a baixa abundância de serpentes. Para verificar se as espécies de répteis da EESGT se distribuem aleatoriamente entre os hábitats amostrados ou se a comunidade apresenta estruturação com relação à distribuição local, foi feita análise de modelos-nulos com o módulo de co-ocorrência do programa EcoSim v.7 (Gotelli \& Entsminger 2008) por meio de 10.000 aleatorizações da matriz original de capturas em cada tipo de hábitat utilizando o índice C-Score, com colunas e linhas fixas, e com a opção "sequencial swap algorithm". Para se observar a similaridade faunística entre os hábitats amostrados foi feita uma análise de agrupamento, utilizando o índice modificado de Morisita-Horn como medida de similaridade faunística e UPGMA como algoritmo de agrupamento, com o programa MVSP v.3.1 (Kovach 2000). Para esta análise, utilizou-se a matriz do número de capturas em cada hábitat somando as capturas manuais durante as buscas ativas e encontros ocasionais e excetuando os indivíduos capturados em coletas manuais intensivas direcionadas (Gymnodactylus amarali e Phyllopezus pollicaris).

Registros de répteis obtidos em inventário prévio dentro dos limites e entorno da EESGT (resultados parciais em Nogueira et al. 2009) e levantamentos na região do Jalapão (Vitt et al. 2005) foram adicionados aos registros obtidos neste estudo para a compilação de uma lista local e outra regional da fauna de répteis do Jalapão. Os padrões de distribuição das espécies na região do Cerrado e em escala continental foram determinados a partir da literatura e padronizados conforme Strüsmann \& Mott (2009). Estes foram comparados com o intuito de observar as relações e contribuições faunísticas de domínios adjacentes para a região do Jalapão. O status de conservação das espécies registradas foi avaliado com consulta a listas de fauna ameaçada de extinção (Martins \& Molina 2008, IUCN 2009) e aos anexos da Convenção sobre o Comércio Internacional de Espécies Ameaçadas (CITES 2009).

\section{Resultados}

Neste estudo registramos um total de 45 espécies de répteis para a EESGT e entorno (Tabela 2, Figura 5), dentre as quais 21 espécies de lagartos, 21 de serpentes, duas de anfisbenídeos e uma de crocodiliano. Computados os estudos prévios realizados na região, têm-se um total de 48 espécies de répteis para a estação e 88 para a região do Jalapão (Tabela 3). Com o presente estudo adicionamos 18 espécies à lista prévia de répteis da EESGT e entorno (Nogueira et al. 2009, C. Nogueira dados não publicados) sendo oito registros inéditos para a região do Jalapão (Tabela 3).

Considerando cada etapa do levantamento separadamente, amostramos 17 espécies de lagartos, duas de anfisbenídeos e 16 de serpentes na porção sul da estação, no Tocantins; e 14 espécies de lagartos, uma de anfisbenídeo e nove de serpentes na porção leste da estação, pertencente ao Chapadão Ocidental da Bahia, sendo o lagarto Cnemidophorus mumbuca registrado pela primeira vez para o Estado da Bahia.

A curva de acumulação de espécies de lagartos e anfisbenas baseada no esforço de coleta para as regiões sul e leste da estação tende a uma assíntota (Figuras 6 e 7) indicando que a riqueza amostrada pode ser próxima à riqueza real. Os estimadores de riqueza para lagartos e anfisbenas recuperaram valores entre 20 e 22 espécies para a porção sul (ICE/Chao2 e Jacknife 2) e entre 17 e 19 na porção leste da EESGT (Chao2 e Jacknife 2) o que se aproxima de nossos resultados. Por outro lado, as curvas de acumulação das espécies de serpentes (Figuras 6 e 7) não apresentam ponto de inflexão para ambas as localidades, indicando que a amostragem está aquém da riqueza real. Estimadores de riqueza recuperaram valores entre 25 e 37 espécies de serpentes para a porção sul (Jacknife 1 e ICE) e entre 17 e 20 (Jacknife 1 e ACE) para a porção leste da estação.

O índice C-Score observado foi maior que a distribuição ao acaso em 10.000 simulações [ $\mathrm{S}=45$; observado: 1,243; simulado (média): $1,152 ; \mathrm{p}<0,001]$, resultado que se manteve mesmo com a retirada das espécies com baixo número de capturas $(\mathrm{N}<4)$ da matriz original [ $\mathrm{S}=20$; observado: 1,221 ; simulado (média): 1,$107 ; \mathrm{p}=0,006]$. Isto indica uma distribuição não-aleatória das espécies pelos hábitats amostrados. A análise de agrupamento (Figura 8) revela uma separação faunística entre os hábitats abertos de interflúvio (cerrado típico, campo arenoso e cerrado rupestre) e os hábitats mésicos (mata de galeria e campos úmidos/veredas). Entre os hábitats de interflúvio, há separação do hábitat campestre dos outros ambientes abertos. Entre os hábitats ripários, a mata, cuja fauna apresenta pouca similaridade com a de outros hábitats, separa-se dos campos úmidos/veredas, caracterizados por apresentarem espécies típicas e compartilhadas com mata, mas principalmente com áreas abertas. A Tabela 2 relaciona os hábitats onde cada espécie foi encontrada.

$\mathrm{Na}$ composição da fauna de répteis da região do Jalapão prevalecem espécies de ampla distribuição geográfica e o compartilhamento de espécies com domínios geomorfológicos vizinhos, sobretudo a Caatinga (Tabela 3). Há também elevado número de espécies típicas de Cerrado, algumas delas endêmicas do Jalapão (Tabela 3). As espécies de répteis presentes na região apresentam três padrões gerais de distribuição: (1) espécies endêmicas do Cerrado representadas por 31 das 88 espécies registradas $(35,2 \%)$, sendo que ao menos cinco $(5,7 \%)$ tem a distribuição conhecida restrita à região do Jalapão; (2) espécies compartilhadas com domínios secos da diagonal de formações abertas sul-americanas, representado por 19 espécies (21,6\%); sendo 9 espécies (10,2\%) compartilhadas exclusivamente com o domínio das Caatingas; (3) espécies de 
Recoder, R.S. et al.

(1)
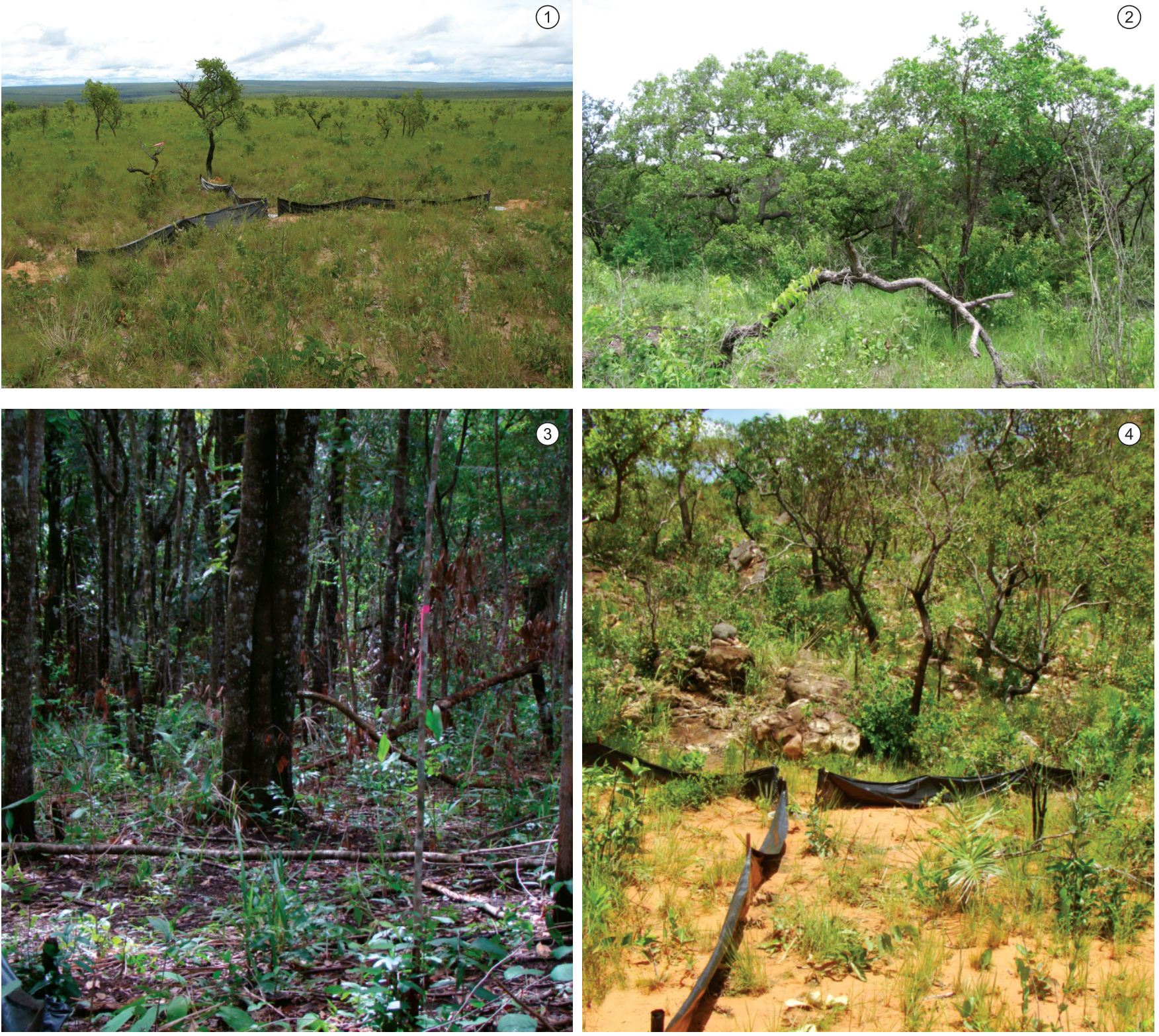

(5)

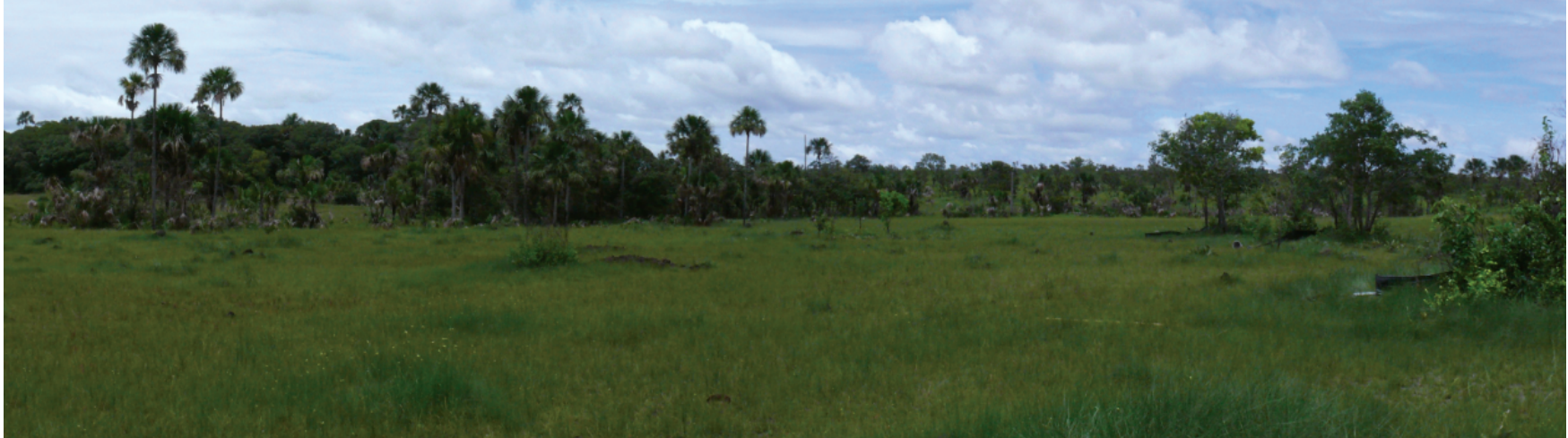

Figura 4. Exemplos de habitats amostrados: 1. Campo sujo; 2. Cerrado típico denso; 3 . Mata de galeria; 4. Cerrado típico aberto, sob influência de encosta de morro; 5. Campo úmido adjacente a vereda. (Fotos: 1 - M. Teixeira Junior; 2 - C.B. Tocchet; 3, 4 - A. Camacho; 5 - R.S. Recoder).

Figure 4. Examples of sampled habitats: 1. Grasslands; 2. Typical savannas; 3. Gallery forest; 4. Open typical savanna, under influence of hill slope; 5 . Wet grassland adjacent to wet marshes. (Photos: 1 - M. Teixeira Junior; 2 - C.B. Tocchet; 3, 4 - A. Camacho; 5 - R.S. Recoder). 
Répteis da EESGT, Brasil Central

Tabela 1. Linhas de pitfalls instaladas nas duas localidades amostradas na EESGT, indicando o esforço de captura e o tipo de hábitat amostrado.

Table 1. Lines of pitfall traps installed in the two sampled sites of EESGT, indicating sampling effort and type of habitat sampled.

\begin{tabular}{|c|c|c|c|c|c|c|}
\hline & $\begin{array}{l}\text { Almas, TO } \\
\text { (esforço) }\end{array}$ & Hábitat & $\begin{array}{c}\text { Coordenadas } \\
\text { (Datum WGS 84) }\end{array}$ & $\begin{array}{c}\text { Formosa do } \\
\text { Rio Preto, BA } \\
\text { (esforço) }\end{array}$ & Hábitat & $\begin{array}{c}\text { Coordenadas } \\
\text { (Datum WGS 84) }\end{array}$ \\
\hline Linha 1 & 160 & Campo sujo & $\begin{array}{l}11^{\circ} 14^{\prime} 51^{\prime \prime} \mathrm{S} \\
46^{\circ} 55^{\prime} 05^{\prime}, \mathrm{W}\end{array}$ & 180 & Campo cerrado & $\begin{array}{l}10^{\circ} 37^{\prime} 08^{\prime \prime} \mathrm{S} \\
46^{\circ} 06^{\prime} 52^{\prime}, \mathrm{W}\end{array}$ \\
\hline Linha 2 & 160 & Campo sujo & $\begin{array}{l}11^{\circ} 18^{\prime} 41^{\prime \prime} \mathrm{S} \\
46^{\circ} 56^{\prime} 06^{\prime}, \mathrm{W}\end{array}$ & 180 & Campo cerrado & $\begin{array}{l}10^{\circ} 37^{\prime} 10^{\prime \prime} \mathrm{S} \\
46^{\circ} 07^{\prime} 10^{\prime \prime} \mathrm{W}\end{array}$ \\
\hline Linha 3 & 160 & Mata de galeria & $\begin{array}{l}11^{\circ} 18^{\prime} 37^{\prime \prime}, \mathrm{S} \\
4956^{\prime} 08^{\prime \prime} \mathrm{W}\end{array}$ & 180 & Cerrado típico & $\begin{array}{l}10^{\circ} 37^{\prime} 29^{\prime \prime} \mathrm{S} \\
46^{\circ} 08^{\prime} 02^{\prime}, \mathrm{W}\end{array}$ \\
\hline Linha 4 & 180 & Campo úmido & $\begin{array}{l}11^{\circ} 13^{\prime} 15^{\prime \prime} \mathrm{S} \\
46^{\circ} 53^{\prime} 08^{\prime}, \mathrm{W}\end{array}$ & 180 & Campo sujo & $\begin{array}{c}10^{\circ} 38^{\prime} 24^{\prime \prime} \mathrm{S} \\
46^{\circ} 09^{\prime} 05^{\prime} \mathrm{W}\end{array}$ \\
\hline Linha 5 & 180 & Campo cerrado & $\begin{array}{l}11^{\circ} 13^{\prime} 15^{\prime}, \mathrm{S} \\
46^{\circ} 53^{\prime} 08^{\prime}, \mathrm{W}\end{array}$ & 180 & Campo sujo & $\begin{array}{l}10^{\circ} 39^{\prime} 40^{\prime \prime} \mathrm{S} \\
46^{\circ} 09^{\prime} 33^{\prime} \mathrm{W}\end{array}$ \\
\hline Linha 6 & 180 & $\begin{array}{c}\text { Cerrado típico em } \\
\text { encosta }\end{array}$ & $\begin{array}{l}11^{\circ} 11^{\prime} 04^{\prime \prime} \mathrm{S} \\
46^{\circ} 50^{\prime} 38^{\prime}, \mathrm{W}\end{array}$ & 180 & $\begin{array}{c}\text { Cerrado } \\
\text { pedregoso }\end{array}$ & $\begin{array}{l}10^{\circ} 40^{\prime} 11^{\prime \prime} \mathrm{S} \\
46^{\circ} 09^{\prime} 06^{\prime} \mathrm{W}\end{array}$ \\
\hline Linha 7 & 180 & Cerrado típico & $\begin{array}{l}11^{\circ} 07^{\prime} 25^{\prime \prime} \mathrm{S} \\
46^{\circ} 47^{\prime} 26^{\prime} \mathrm{W}\end{array}$ & 180 & $\begin{array}{c}\text { Cerrado típico em } \\
\text { encosta }\end{array}$ & $\begin{array}{c}10^{\circ} 40^{\prime} 48^{\prime \prime} \mathrm{S} \\
46^{\circ} 09^{\prime} 02^{\prime} \mathrm{W}\end{array}$ \\
\hline Linha 8 & 180 & $\begin{array}{c}\text { Cerrado típico em } \\
\text { encosta }\end{array}$ & $\begin{array}{l}11^{\circ} 07^{\prime} 12^{\prime \prime}, \mathrm{S} \\
46^{\circ} 47^{\prime} 12^{\prime}, \mathrm{W}\end{array}$ & 180 & Campo limpo & $\begin{array}{l}10^{\circ} 44^{\prime} 12^{\prime \prime} \mathrm{S} \\
46^{\circ} 11^{\prime} 48^{\prime}, \mathrm{W}\end{array}$ \\
\hline Linha 9 & 180 & Cerrado típico & $\begin{array}{l}11^{\circ} 06^{\prime} 07^{\prime}, \mathrm{S} \\
46^{\circ} 45^{\prime} 57^{\prime}, \mathrm{W}\end{array}$ & 180 & Campo limpo & $\begin{array}{l}10^{\circ} 43^{\prime} 32^{\prime}, \mathrm{S} \\
46^{\circ} 12^{\prime} 45^{\prime}, \mathrm{W}\end{array}$ \\
\hline Total & \multicolumn{3}{|c|}{1560 pitfalls $\times$ dias } & 1620 pitfalls $\times d$ & & \\
\hline
\end{tabular}

Tabela 2. Uso de hábitat das espécies amostradas na EESGT. Números representam indivíduos capturados com armadilhas de interceptação e queda e números entre parênteses representam indivíduos capturados manualmente.

Table 2. Use of habitat of the species sampled in EESGT. Numbers represents individuals captured in pitfall traps and numbers between brackets represents individuals captured by hand.

\begin{tabular}{|c|c|c|c|c|c|c|}
\hline & $\mathbf{N}$ & $\begin{array}{l}\text { Cerrado } \\
\text { rupestre }\end{array}$ & $\begin{array}{c}\text { Cerrado } \\
\text { típico }\end{array}$ & $\begin{array}{c}\text { Campos } \\
\text { arenosos }\end{array}$ & $\begin{array}{c}\text { Vereda/Campo } \\
\text { úmido } \\
\end{array}$ & $\begin{array}{c}\text { Mata de } \\
\text { galeria }\end{array}$ \\
\hline \multicolumn{7}{|l|}{ LEPIDOSAURIA } \\
\hline \multicolumn{7}{|l|}{ SQUAMATA } \\
\hline \multicolumn{7}{|l|}{ Hoplocercidae } \\
\hline Hoplocercus spinosus & 2 & (1) & 1 & - & - & - \\
\hline \multicolumn{7}{|l|}{ Iguanidae } \\
\hline Iguana iguana & 3 & - & - & - & (1) & (2) \\
\hline \multicolumn{7}{|l|}{ Tropiduridae } \\
\hline Tropidurus cf. oreadicus & 64 & (5) & $12(8)$ & $25(10)$ & $2(2)$ & - \\
\hline Stenocercus quinarius & 1 & - & 1 & - & - & - \\
\hline \multicolumn{7}{|l|}{ Polychrotidae } \\
\hline Anolis chrysolepis & 5 & - & - & - & - & $2(3)$ \\
\hline Polychrus acutirostris & 1 & - & - & - & (1) & - \\
\hline \multicolumn{7}{|l|}{ Sphaerodactylidae } \\
\hline Coleodactylus brachystoma & 25 & (9) & $9(7)$ & - & - & - \\
\hline \multicolumn{7}{|l|}{ Phyllodactylidae } \\
\hline Gymnodactylus amarali & 82 & $(24)$ & (1) & - & $5(52)$ & - \\
\hline Phyllopezus pollicaris & 33 & $(32)$ & (1) & - & - & - \\
\hline \multicolumn{7}{|l|}{ Gekkonidae } \\
\hline Hemidactylus brasilianus & 8 & (1) & 4 & $1(2)$ & - & - \\
\hline Scincidae & & & & & & \\
\hline
\end{tabular}


Tabela 2. Continuação...

\begin{tabular}{|c|c|c|c|c|c|c|}
\hline & $\mathbf{N}$ & $\begin{array}{l}\text { Cerrado } \\
\text { rupestre }\end{array}$ & $\begin{array}{c}\text { Cerrado } \\
\text { típico }\end{array}$ & $\begin{array}{c}\text { Campos } \\
\text { arenosos }\end{array}$ & $\begin{array}{c}\text { Vereda/Campo } \\
\text { úmido }\end{array}$ & $\begin{array}{c}\text { Mata de } \\
\text { galeria }\end{array}$ \\
\hline Mabuya cf. heathi & 15 & - & $4(2)$ & $7(2)$ & - & - \\
\hline Mabuya cf. nigropunctata & 12 & $1(2)$ & $2(1)$ & - & (5) & (1) \\
\hline Mabuya frenata & 1 & - & - & - & - & (1) \\
\hline \multicolumn{7}{|l|}{ Gymnophthalmidae } \\
\hline Bachia oxyrhina & 6 & - & $3(1)$ & $1(1)$ & - & - \\
\hline Cercosaura ocellata & 4 & - & - & - & (4) & - \\
\hline Colobosaura modesta & 6 & - & $4(1)$ & - & (1) & - \\
\hline Micrablepharus maximiliani & 14 & - & $9(1)$ & 4 & - & - \\
\hline Vanzosaura rubricauda & 26 & - & $7(2)$ & $16(1)$ & - & - \\
\hline \multicolumn{7}{|l|}{ Teiidae } \\
\hline Ameiva ameiva & 10 & - & $5(2)$ & $2(1)$ & - & - \\
\hline Cnemidophorus mumbuca & 35 & - & 5 & $29(1)$ & - & - \\
\hline Cnemidophorus jalapensis & 38 & (5) & $1(2)$ & $25(4)$ & - & 1 \\
\hline \multicolumn{7}{|l|}{ Amphisbaenidae } \\
\hline Amphisbaena vermicularis & 1 & - & - & - & - & (1) \\
\hline Amphisbaena leeseri & 7 & $1(2)$ & (2) & (1) & (1) & - \\
\hline \multicolumn{7}{|l|}{ Serpentes } \\
\hline \multicolumn{7}{|l|}{ Leptotyphlopidae } \\
\hline Siagonodon acutirostris & 1 & - & 1 & - & - & - \\
\hline \multicolumn{7}{|l|}{ Typhlopidae } \\
\hline Typhlops brongersmianus & 1 & - & - & 1 & - & - \\
\hline \multicolumn{7}{|l|}{ Boidae } \\
\hline Epicrates crassus & 5 & - & - & (3) & - & $(2)$ \\
\hline Eunectes murinus & 1 & - & - & - & (1) & - \\
\hline \multicolumn{7}{|l|}{ Colubridae } \\
\hline Drymarchon corais & 1 & - & - & - & (1) & - \\
\hline Oxybelis aeneus & 1 & - & - & (1) & - & - \\
\hline \multicolumn{7}{|l|}{ Dipsadidae } \\
\hline Apostolepis nelsonjorgei & 1 & - & 1 & - & - & - \\
\hline Atractus pantostictus & 1 & - & - & - & - & (1) \\
\hline Boiruna cf. sertaneja & 1 & - & - & (1) & - & - \\
\hline Helicops leopardinus & 1 & - & - & - & - & (1) \\
\hline Leptodeira annulata & 1 & - & - & - & - & (1) \\
\hline Oxyrhopus trigeminus & 5 & (1) & 1 & $(2)$ & (1) & - \\
\hline Philodryas nattereri & 3 & - & (2) & - & (1) & - \\
\hline Phimophis iglesiasi & 1 & - & - & (1) & - & - \\
\hline Sibynomorphus mikani & 1 & - & - & - & - & (1) \\
\hline Tantilla melanocephala & 3 & (1) & 1 & 1 & - & - \\
\hline Thamnodynastes hypoconia & 2 & - & - & - & (1) & (1) \\
\hline Xenodon merremi & 1 & - & - & (1) & - & - \\
\hline \multicolumn{7}{|l|}{ Elapidae } \\
\hline Micrurus brasiliensis & 1 & - & - & (1) & - & - \\
\hline \multicolumn{7}{|l|}{ Viperidae } \\
\hline Bothrops moojeni & 6 & - & - & - & (4) & (2) \\
\hline Bothropoides lutzi & 3 & - & - & (3) & - & - \\
\hline \multicolumn{7}{|l|}{ ARCHOSAURIA } \\
\hline \multicolumn{7}{|l|}{ CROCODYLIA } \\
\hline \multicolumn{7}{|l|}{ Alligatoridae } \\
\hline Paleosuchus palpebrosus & 2 & - & - & - & (1) & (1) \\
\hline
\end{tabular}



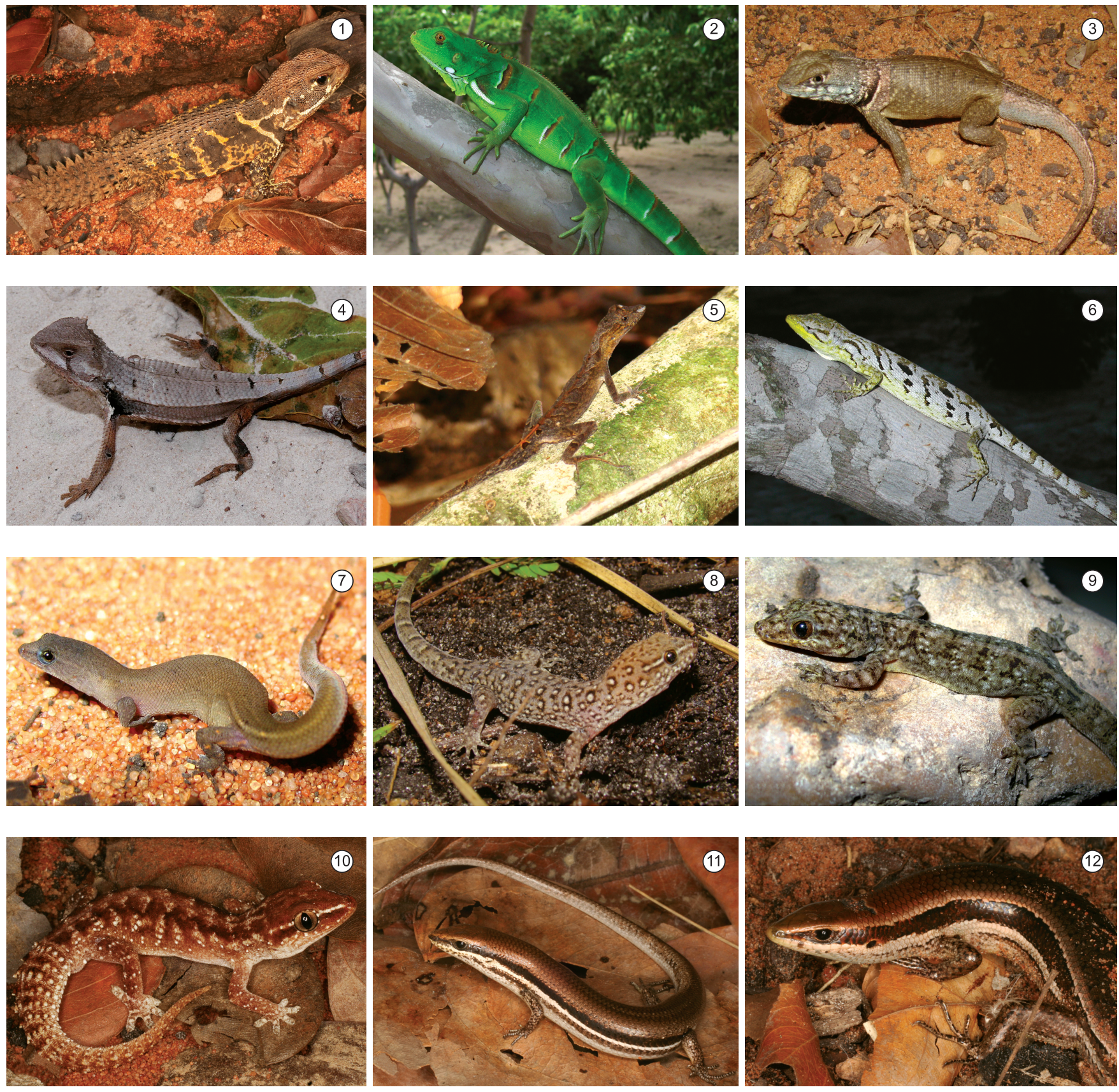

Figura 5. Algumas das espécies de répteis amostradas na EESGT e entorno: 1. Hoplocercus spinosus; 2. Iguana iguana; 3. Tropidurus cf. oreadicus; 4. Stenocercus quinarius; 5. Anolis chrysolepis; 6. Polychrus acutirostris; 7. Coleodactylus brachystoma; 8. Gymnodactylus amarali; 9. Phyllopezus pollicaris; 10. Hemidactylus brasilianus; 11. Mabuya cf. heathi; 12. Mabuya cf. nigropunctata; ...

(Fotos: 1, 10-12, 14, 15, 18, 21, 24, 38, 41, 42 - M.T. Rodrigues; 2, 6, 7, 9, 17, 20, 26, 44, 45 - M. Teixeira Jr.; 3, 5, 8, 13, 16, 23, 25, 29-31, 33, 35, 36, 39, 47 - A. Camacho; 4, 19, 43 - R.S. Recoder; 27, 28, 34, 40 - P.M.S. Nunes; 46 - P.H. Valdujo; 22, 32, 37 - C.C. Nogueira).

Figure 5. Some of the reptile species recorded in EESGT and surrounding area: 1. Hoplocercus spinosus; 2. Iguana iguana; 3. Tropidurus cf. oreadicus; 4. Stenocercus quinarius; 5. Anolis chrysolepis; 6. Polychrus acutirostris; 7. Coleodactylus brachystoma; 8. Gymnodactylus amarali; 9. Phyllopezus pollicaris; 10. Hemidactylus brasilianus; 11. Mabuya cf. heathi; 12. Mabuya cf. nigropunctata; ...

(Photos: 1, 10-12, 14, 15, 18, 21, 24, 38, 41, 42 - M.T. Rodrigues; 2, 6, 7, 9, 17, 20, 26, 44, 45 - M. Teixeira Jr.; 3, 5, 8, 13, 16, 23, 25, 29-31, 33, 35, 36, 39, 47 - A. Camacho; 4, 19, 43 - R.S. Recoder; 27, 28, 34, 40 - P.M.S. Nunes; 46 - P.H. Valdujo; 22, 32, 37 - C.C. Nogueira). 

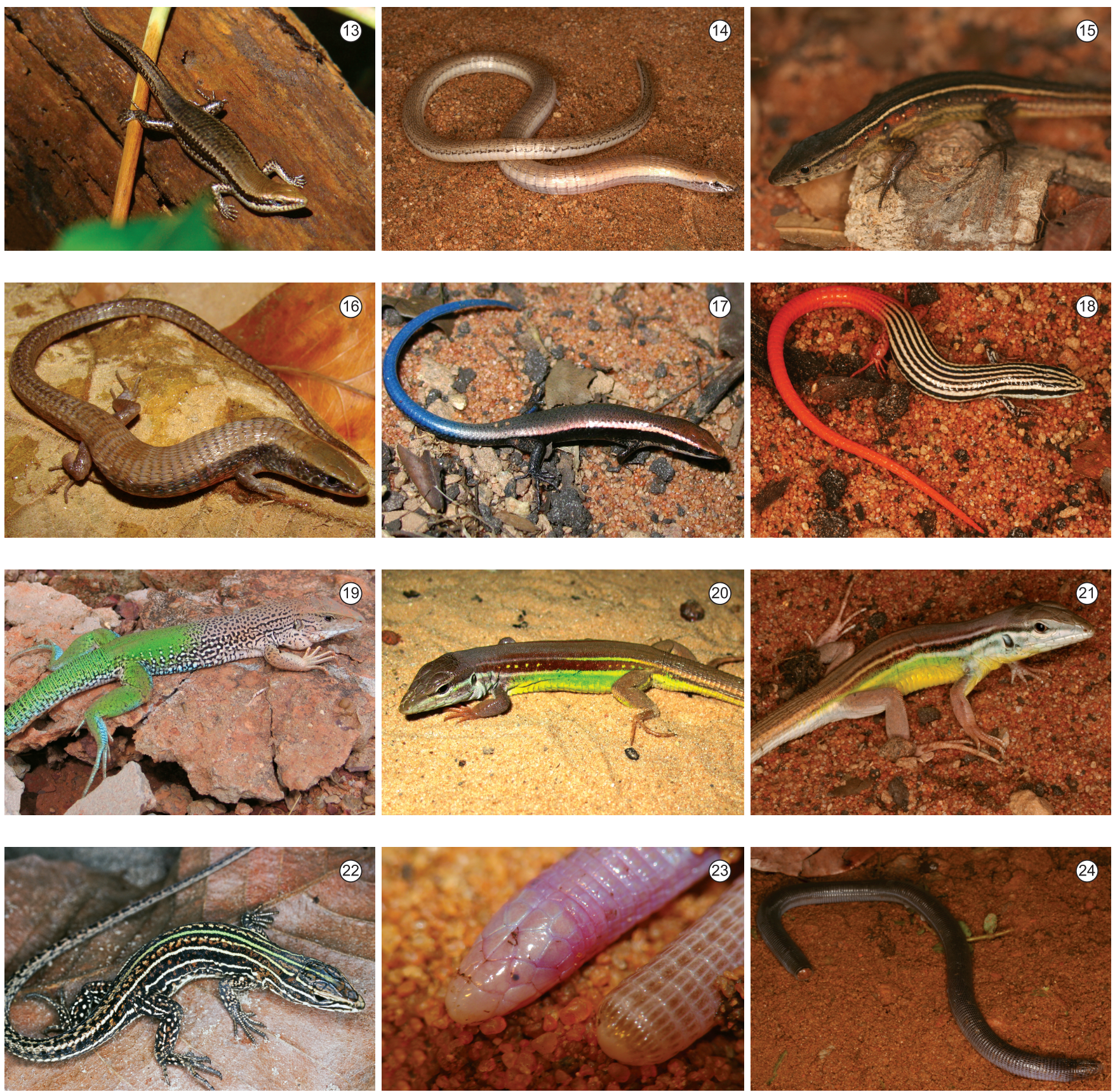

Figura 5. ... 13. Mabuya frenata; 14. Bachia oxyrhina; 15. Cercosaura ocellata; 16. Colobosaura modesta; 17. Micrablepharus maximiliani; 18. Vanzosaura rubricauda; 19. Ameiva ameiva; 20. Cnemidophorus mumbuca; 21. Cnemidophorus jalapensis; 22. Kentropyx aff. paulensis 23. Amphisbaena leeseri; 24. A. vermicularis; ...

(Fotos: 1, 10-12, 14, 15, 18, 21, 24, 38, 41, 42 - M.T. Rodrigues; 2, 6, 7, 9, 17, 20, 26, 44, 45 - M. Teixeira Jr.; 3, 5, 8, 13, 16, 23, 25, 29-31, 33, 35, 36, 39, 47 - A. Camacho; 4, 19, 43 - R.S. Recoder; 27, 28, 34, 40 - P.M.S. Nunes; 46 - P.H. Valdujo; 22, 32, 37 - C.C. Nogueira).

Figure 5. ... 13. Mabuya frenata; 14. Bachia oxyrhina; 15. Cercosaura ocellata; 16. Colobosaura modesta; 17. Micrablepharus maximiliani; 18. Vanzosaura rubricauda; 19. Ameiva ameiva; 20. Cnemidophorus mumbuca; 21. Cnemidophorus jalapensis; 22. Kentropyx aff. paulensis 23. Amphisbaena leeseri; 24. A. vermicularis; ...

(Photos: 1, 10-12, 14, 15, 18, 21, 24, 38, 41, 42 - M.T. Rodrigues; 2, 6, 7, 9, 17, 20, 26, 44, 45 - M. Teixeira Jr.; 3, 5, 8, 13, 16, 23, 25, 29-31, 33, 35, 36, 39, 47 - A. Camacho; 4, 19, 43 - R.S. Recoder; 27, 28, 34, 40 - P.M.S. Nunes; 46 - P.H. Valdujo; 22, 32, 37 - C.C. Nogueira). 

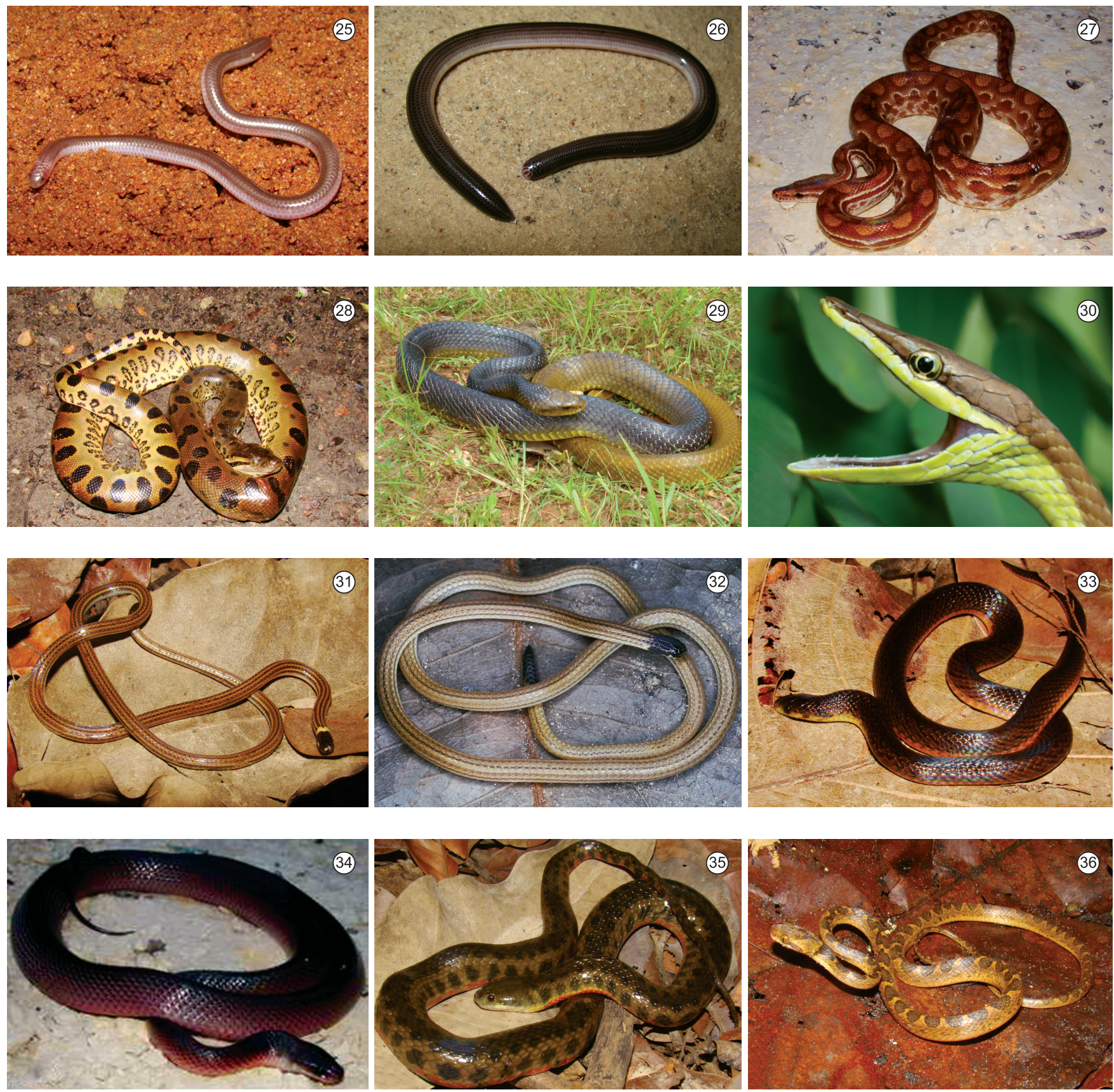

Figura 5. ... 25. Siagonodon acutirostris; 26. Typhlops brongersmianus; 27. Epicrates crassus; 28. Eunectes murinus; 29. Drymarchon corais; 30. Oxybelis aeneus; 31 . Apostolepis nelsonjorgei; 32. Apostolepis aff. longicaudata; 33. Atractus pantostictus; 34. Boiruna cf. sertaneja; 35 . Helicops leopardinus; 36. Leptodeira annulata; ...

(Fotos: 1, 10-12, 14, 15, 18, 21, 24, 38, 41, 42 - M.T. Rodrigues; 2, 6, 7, 9, 17, 20, 26, 44, 45 - M. Teixeira Jr.; 3, 5, 8, 13, 16, 23, 25, 29-31, 33, 35, 36, 39, 47 - A. Camacho; 4, 19, 43 - R.S. Recoder; 27, 28, 34, 40 - P.M.S. Nunes; 46 - P.H. Valdujo; 22, 32, 37 - C.C. Nogueira).

Figure 5. ... 25. Siagonodon acutirostris; 26. Typhlops brongersmianus; 27. Epicrates crassus; 28. Eunectes murinus; 29. Drymarchon corais; 30. Oxybelis aeneus; 31. Apostolepis nelsonjorgei; 32. Apostolepis aff. longicaudata; 33. Atractus pantostictus; 34. Boiruna cf. sertaneja; 35. Helicops leopardinus; 36. Leptodeira annulata; ...

(Photos: 1, 10-12, 14, 15, 18, 21, 24, 38, 41, 42 - M.T. Rodrigues; 2, 6, 7, 9, 17, 20, 26, 44, 45 - M. Teixeira Jr.; 3, 5, 8, 13, 16, 23, 25, 29-31, 33, 35, 36, 39, 47 - A. Camacho; 4, 19, 43 - R.S. Recoder; 27, 28, 34, 40 - P.M.S. Nunes; 46 - P.H. Valdujo; 22, 32, 37 - C.C. Nogueira). 

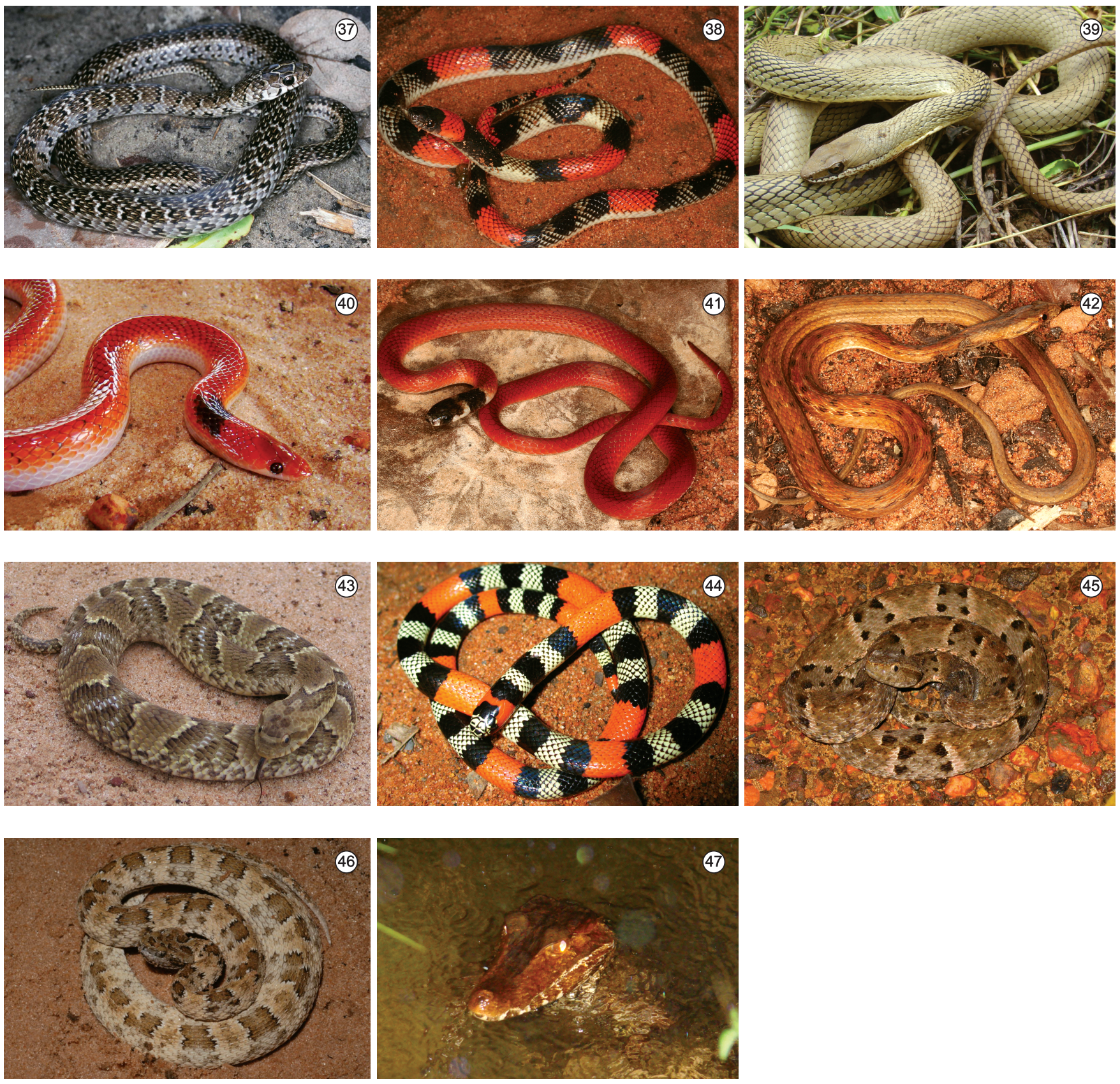

Figura 5. ... 37. Liophis almadensis; 38. Oxyrhopus trigeminus; 39. Philodryas nattereri; 40. Phimophis iglesiasi; 41. Tantilla melanocephala; 42. Thamnodynastes hypoconia; 43. Xenodon merremi; 44. Micrurus brasiliensis; 45. Bothrops moojeni; 46. Bothropoides lutzi; 47. Paleosuchus palpebrosus. (Fotos: 1, 10-12, 14, 15, 18, 21, 24, 38, 41, 42 - M.T. Rodrigues; 2, 6, 7, 9, 17, 20, 26, 44, 45 - M. Teixeira Jr.; 3, 5, 8, 13, 16, 23, 25, 29-31, 33, 35, 36, 39, 47 - A. Camacho; 4, 19, 43 - R.S. Recoder; 27, 28, 34, 40 - P.M.S. Nunes; 46 - P.H. Valdujo; 22, 32, 37 - C.C. Nogueira).

Figure 5. ... 37. Liophis almadensis; 38. Oxyrhopus trigeminus; 39. Philodryas nattereri; 40. Phimophis iglesiasi; 41. Tantilla melanocephala; 42. Thamnodynastes hypoconia; 43. Xenodon merremi; 44. Micrurus brasiliensis; 45. Bothrops moojeni; 46. Bothropoides lutzi; 47. Paleosuchus palpebrosus. (Photos: 1, 10-12, 14, 15, 18, 21, 24, 38, 41, 42 - M.T. Rodrigues; 2, 6, 7, 9, 17, 20, 26, 44, 45 - M. Teixeira Jr.; 3, 5, 8, 13, 16, 23, 25, 29-31, 33, 35, 36, 39, 47 - A. Camacho; 4, 19, 43 - R.S. Recoder; 27, 28, 34, 40 - P.M.S. Nunes; 46 - P.H. Valdujo; 22, 32, 37 - C.C. Nogueira). 
Tabela 3. Distribuição local, distribuição no Cerrado e ocorrência geográfica das espécies de répteis registradas para a região do Jalapão. Registros para a EESGT: TO = Tocantins, BA = Bahia. Em negrito: registros novos para a EESGT. Padrão de distribuição no Cerrado: AO - ampla ocorrência; M - marginal; R - restrita. "E" sobrescrito indica espécies endêmicas da região. Ocorrência: CE - Cerrado; CA - Caatinga; MA - Mata Atlântica; PA - Pampas; AM - Amazônia; CH - Chaco. Sobrescrito no nome das espécies está o status de conservação: CITES (anexo I a III); LC - "least concern" (IUCN 2009).

Table 3. Local distribution, distributional pattern in the Cerrado and geographical distribution of reptile species recorded for the Jalapão region. Records to EESGT: TO = Tocantins, BA = Bahia. In bold: new records for the EESGT. Distributional patterns in the Cerrado: AO - widespread, M - peripheral; R - restricted. "E" superscripted indicates species endemic to Jalapão region. Geographical occurrence: CE - Cerrados, CA - Caatinga, MA - Atlantic Forest, PA - Pampas grasslands; AM - Amazonia, CH - Chaco. Superscript in the species names are the conservation status: CITES (Annexes I to III), LC - least concern (IUCN 2009).

$\begin{array}{cccc}\text { EESGT } & \begin{array}{c}\text { Registros prévios para } \\ \text { a região do Jalapão } \\ \text { (Vitt et al. 2005) }\end{array} & \begin{array}{c}\text { Distribuição } \\ \text { no Cerrado }\end{array} & \begin{array}{c}\text { Distribuição no } \\ \text { continente }\end{array} \\ & & \end{array}$

\begin{tabular}{|c|c|c|c|c|}
\hline \multicolumn{5}{|l|}{ LEPIDOSAURIA } \\
\hline \multicolumn{5}{|l|}{ SQUAMATA } \\
\hline \multicolumn{5}{|l|}{ Hoplocercidae } \\
\hline Hoplocercus spinosus Fitzinger, 1843 & TO & $\mathrm{X}$ & $\mathrm{AO}$ & $\mathrm{CE}$ \\
\hline \multicolumn{5}{|l|}{ Iguanidae } \\
\hline Iguana iguana (Linnaeus, 1758) (ITES II $^{\text {CI }}$ & TO, BA & $\mathrm{X}$ & $\mathrm{AO}$ & $\mathrm{CE}, \mathrm{CA}, \mathrm{AM}, \mathrm{MA}$ \\
\hline \multicolumn{5}{|l|}{ Tropiduridae } \\
\hline Tropidurus cf. oreadicus Rodrigues, 1987 & TO, BA & $\mathrm{X}$ & $\mathrm{AO}$ & $\mathrm{CE}$ \\
\hline Stenocercus quinarius Nogueira \& Rodrigues, 2006 & BA & - & $\mathrm{R}$ & $\mathrm{CE}$ \\
\hline \multicolumn{5}{|l|}{ Polychrotidae } \\
\hline Anolis chrysolepis Duméril \& Bibron, 1837 & TO & $X$ & $\mathrm{AO}$ & $\mathrm{CE}, \mathrm{AM}$ \\
\hline Polychrus acutirostris Spix, 1825 & TO, BA & $\mathrm{X}$ & $\mathrm{AO}$ & $\mathrm{CE}, \mathrm{CA}, \mathrm{CH}$ \\
\hline \multicolumn{5}{|l|}{ Sphaerodactylidae } \\
\hline Coleodactylus brachystoma (Amaral, 1935) & TO, BA & & $\mathrm{AO}$ & $\mathrm{CE}$ \\
\hline Coleodactylus cf. meridionalis (Boulenger, 1888) & - & $\mathrm{X}$ & $\mathrm{R}$ & CE, CA, MA \\
\hline \multicolumn{5}{|l|}{ Phyllodactylidae } \\
\hline Gymnodactylus amarali Barbour, 1925 & TO & $\mathrm{X}$ & $\mathrm{AO}$ & $\mathrm{CE}$ \\
\hline Phyllopezus pollicaris (Spix, 1825) & TO, BA & - & $\mathrm{AO}$ & $\mathrm{CE}, \mathrm{CA}, \mathrm{CH}, \mathrm{MA}$ \\
\hline \multicolumn{5}{|l|}{ Gekkonidae } \\
\hline Hemidactylus brasilianus (Amaral, 1935) & TO, BA & $\mathrm{X}$ & M & $\mathrm{CE}, \mathrm{CA}$ \\
\hline Hemidactylus mabouia (Moreau de Jonnès, 1818) & TO & $\mathrm{X}$ & $\mathrm{AO}$ & Periantrópico \\
\hline \multicolumn{5}{|l|}{ Scincidae } \\
\hline Mabuya cf. heathi Schmidt \& Inger, 1951 & TO, BA & $\mathrm{X}$ & M & $\mathrm{CE}, \mathrm{CA}$ \\
\hline Mabuya cf. nigropunctata (Spix, 1825) & TO, BA & $\mathrm{X}$ & $\mathrm{AO}$ & $\mathrm{CE}, \mathrm{AM}, \mathrm{MA}$ \\
\hline Mabuya frenata (Cope, 1862) & TO & - & $\mathrm{AO}$ & $\mathrm{CE}, \mathrm{CH}, \mathrm{MA}$ \\
\hline \multicolumn{5}{|l|}{ Gymnophthalmidae } \\
\hline Bachia oxyrhina Rodrigues et al., 2008 & TO & - & $\mathrm{R}^{\mathrm{E}}$ & $\mathrm{CE}$ \\
\hline Cercosaura ocellata Wagler, 1830 & TO & $\mathrm{X}$ & $\mathrm{AO}$ & CE, AM, MA \\
\hline Colobosaura modesta (Reinhardt \& Lütken, 1862) & $\mathrm{TO}, \mathbf{B A}$ & $\mathrm{X}$ & $\mathrm{AO}$ & $\mathrm{CE}$ \\
\hline Micrablepharus maximiliani (Reinhardt \& Lütken, 1862) & TO, BA & $\mathrm{X}$ & $\mathrm{AO}$ & $\mathrm{CE}, \mathrm{CA}, \mathrm{MA}$ \\
\hline Vanzosaura rubricauda (Boulenger, 1902) & TO, BA & $\mathrm{X}$ & $\mathrm{AO}$ & $\mathrm{CE}, \mathrm{CA}, \mathrm{CH}$ \\
\hline \multicolumn{5}{|l|}{ Teiidae } \\
\hline Ameiva ameiva (Linnaeus, 1758) & TO, BA & $\mathrm{X}$ & $\mathrm{AO}$ & $\begin{array}{c}\mathrm{CE}, \mathrm{CA}, \mathrm{CH}, \mathrm{AM}, \\
\mathrm{MA}\end{array}$ \\
\hline Cnemidophorus mumbuca Colli et al., 2003 & $\mathrm{TO}, \mathbf{B A}$ & $\mathrm{X}$ & $\mathrm{R}$ & $\mathrm{CE}$ \\
\hline Cnemidophorus jalapensis Colli et al., 2009 & TO & $\mathrm{X}$ & $\mathrm{R}^{\mathrm{E}}$ & $\mathrm{CE}$ \\
\hline Kentropyx aff. paulensis Boettger, 1893 & TO & $\mathrm{X}$ & $\mathrm{R}^{\mathrm{E}}$ & $\mathrm{CE}$ \\
\hline Tupinambis duseni Lönnberg, 1910 & - & $\mathrm{X}$ & $\mathrm{AO}$ & $\mathrm{CE}$ \\
\hline Tupinambis merianae (Duméril \& Bibron, 1839) & - & $\mathrm{X}$ & $\mathrm{AO}$ & $\begin{array}{c}\mathrm{CE}, \mathrm{CA}, \mathrm{CH}, \mathrm{MA}, \\
\mathrm{PA}, \mathrm{AM}\end{array}$ \\
\hline Tupinambis quadrilineatus Manzani \& Abe, $1997^{\text {CITES II }}$ & - & $\mathrm{X}$ & $\mathrm{AO}$ & $\mathrm{CE}$ \\
\hline Amphisbaenidae & & & & \\
\hline
\end{tabular}




\begin{tabular}{|c|c|c|c|c|}
\hline & EESGT & $\begin{array}{l}\text { Registros prévios para } \\
\text { a região do Jalapão } \\
\text { (Vitt et al. 2005) }\end{array}$ & $\begin{array}{l}\text { Distribuição } \\
\text { no Cerrado }\end{array}$ & $\begin{array}{l}\text { Distribuição no } \\
\text { continente }\end{array}$ \\
\hline $\begin{array}{l}\text { Amphisbaena acrobeles (Ribeiro, Castro-Mello \& } \\
\text { Nogueira, 2009) }\end{array}$ & TO & - & $\mathrm{R}^{\mathrm{E}}$ & $\mathrm{CE}$ \\
\hline Amphisbaena alba Linnaeus, 1758 & TO & $\mathrm{X}$ & $\mathrm{AO}$ & CE, AM, MA \\
\hline Amphisbaena kraoh (Vanzolini, 1971) & - & $\mathrm{X}$ & $\mathrm{R}$ & $\mathrm{CE}$ \\
\hline Amphisbaena leeseri Gans, 1964 & TO, BA & $\mathrm{X}$ & $\mathrm{R}$ & $\mathrm{CE}$ \\
\hline Amphisbaena polystega (Duméril, 1851) & - & $\mathrm{X}$ & $\mathrm{M}$ & $\mathrm{CE}, \mathrm{CA}$ \\
\hline Amphisbaena vermicularis Wagler, 1824 & TO & & $\mathrm{AO}$ & $\mathrm{CE}, \mathrm{CA}, \mathrm{AM}$ \\
\hline \multicolumn{5}{|l|}{ Serpentes } \\
\hline \multicolumn{5}{|l|}{ Leptotyphlopidae } \\
\hline Siagonodon acutirostris Pinto \& Curcio, 2011 & TO & $\mathrm{X}$ & $\mathrm{R}$ & $\mathrm{CE}$ \\
\hline \multicolumn{5}{|l|}{ Typhlopidae } \\
\hline Typhlops brongersmianus Vanzolini, 1976 & TO, BA & $\mathrm{X}$ & $\mathrm{AO}$ & $\mathrm{CE}, \mathrm{CH}, \mathrm{AM}, \mathrm{MA}$ \\
\hline \multicolumn{5}{|l|}{ Boidae } \\
\hline Boa constrictor Linnaeus, $1758^{\text {CITES II }}$ & - & $\mathrm{X}$ & $\mathrm{AO}$ & $\begin{array}{l}\mathrm{CE}, \mathrm{CA}, \mathrm{CH}, \mathrm{AM}, \\
\text { MA }\end{array}$ \\
\hline Corallus hortulanus (Linnaeus, 1758) CITES II $^{\text {II }}$ & - & $\mathrm{X}$ & $\mathrm{AO}$ & $\mathrm{CE}, \mathrm{CA}, \mathrm{AM}, \mathrm{MA}$ \\
\hline Epicrates crassus Cope, $1862^{\mathrm{CITES} \text { II }}$ & TO, BA & $\mathrm{X}$ & $\mathrm{AO}$ & $\mathrm{CE}$ \\
\hline Eunectes murinus (Linnaeus, 1758) & - & $\mathrm{X}$ & $\mathrm{AO}$ & $\mathrm{CE}, \mathrm{CH}, \mathrm{AM}, \mathrm{CA}$ \\
\hline \multicolumn{5}{|l|}{ Colubridae } \\
\hline Chironius flavolineatus (Boettger, 1885) & - & $\mathrm{X}$ & $\mathrm{AO}$ & $\mathrm{CE}, \mathrm{CA}, \mathrm{CH}$ \\
\hline Chironius quadricarinatus (Boie, 1827) & - & $\mathrm{X}$ & $\mathrm{AO}$ & $\mathrm{CE}$ \\
\hline Drymarchon corais (Boie, 1827$)$ & TO & $\mathrm{X}$ & $\mathrm{AO}$ & $\mathrm{CE}, \mathrm{CA}, \mathrm{AM}, \mathrm{MA}$ \\
\hline Mastigodryas bifossatus (Raddi, 1820) & - & $\mathrm{X}$ & $\mathrm{AO}$ & $\begin{array}{l}\mathrm{CE}, \mathrm{CA}, \mathrm{CH}, \mathrm{AM}, \\
\text { MA, PA }\end{array}$ \\
\hline Mastigodryas boddaertti (Sentzen, 1796) & - & $\mathrm{X}$ & $\mathrm{R}$ & $\mathrm{CE}, \mathrm{AM}$ \\
\hline Oxybelis aeneus (Wagler, 1824) & TO & $\mathrm{X}$ & $\mathrm{AO}$ & $\mathrm{CE}, \mathrm{CA}, \mathrm{AM}, \mathrm{MA}$ \\
\hline \multicolumn{5}{|l|}{ Dipsadidae } \\
\hline $\begin{array}{l}\text { Apostolepis ammodites Ferrarezzi, Barbo \& Albuquerque, } \\
2005\end{array}$ & TO & & $\mathrm{AO}$ & $\mathrm{CE}$ \\
\hline Apostolepis cf. longicaudata Amaral, 1921 & TO & & $\mathrm{R}$ & $\mathrm{CE}$ \\
\hline Apostolepis nelsonjorgei Lema \& Renner 2004 & TO & & $\mathrm{R}$ & $\mathrm{CE}$ \\
\hline Apostolepis polylepis Amaral, 1921 & - & $\mathrm{X}$ & $\mathrm{R}$ & $\mathrm{CE}$ \\
\hline Atractus pantostictus Fernandes \& Puorto, 1993 & TO & - & $\mathrm{AO}$ & CE, MA \\
\hline Boiruna cf. sertaneja Zaher 1996 & TO, BA & $\mathrm{X}$ & M & $\mathrm{CE}, \mathrm{CA}$ \\
\hline Clelia plumbea $($ Wied 1820$)$ & - & $\mathrm{X}$ & $\mathrm{AO}$ & $\mathrm{CE}, \mathrm{CA}, \mathrm{AM}, \mathrm{MA}$ \\
\hline Helicops angulatus (Linnaeus, 1758) & - & $\mathrm{X}$ & $\mathrm{AO}$ & CE, AM, MA \\
\hline Helicops leopardinus (Schlegel, 1837) & TO & $\mathrm{X}$ & $\mathrm{AO}$ & $\mathrm{CE}, \mathrm{CA}, \mathrm{CH}, \mathrm{AM}$ \\
\hline Hydrodynastes gigas (Duméril, Bibron \& Duméril, 1854) & - & $\mathrm{X}$ & $\mathrm{AO}$ & $\mathrm{CE}, \mathrm{CH}, \mathrm{AM}, \mathrm{MA}$ \\
\hline Leptodeira annulata (Linnaeus, 1758) & - & $\mathrm{X}$ & $\mathrm{AO}$ & $\begin{array}{l}\mathrm{CE}, \mathrm{CA}, \mathrm{CH}, \mathrm{AM}, \\
\text { MA }\end{array}$ \\
\hline Liophis almadensis (Wagler, 1824) & - & $\mathrm{X}$ & $\mathrm{AO}$ & $\mathrm{CE}, \mathrm{CA}, \mathrm{CH}, \mathrm{MA}$ \\
\hline Liophis cf. frenatus (Werner, 1909) & - & $\mathrm{X}$ & $\mathrm{R}$ & $\mathrm{CE}$ \\
\hline Liophis maryellenae Dixon, 1985 & - & $\mathrm{X}$ & $\mathrm{AO}$ & $\mathrm{CE}$ \\
\hline Liophis reginae (Linnaeus, 1758) & - & $\mathrm{X}$ & $\mathrm{AO}$ & $\mathrm{CE}, \mathrm{CA}, \mathrm{AM}$ \\
\hline Lygophis meridionalis (Schenkel, 1902) & - & $\mathrm{X}$ & $\mathrm{AO}$ & $\mathrm{CE}$ \\
\hline Lygophis paucidens Hoge, 1953 & - & $\mathrm{X}$ & $\mathrm{AO}$ & $\mathrm{CE}$ \\
\hline Oxyrhopus guibei Hoge \& Romano, 1977 & - & $\mathrm{X}$ & $\mathrm{AO}$ & CE, MA \\
\hline Oxyrhopus rhombifer Duméril, Bibron \& Duméril, 1854 & - & $\mathrm{X}$ & $\mathrm{AO}$ & $\mathrm{CE}, \mathrm{CH}, \mathrm{PA}$ \\
\hline Oxyrhopus trigeminus Duméril, Bibron \& Duméril, 1854 & TO, BA & $\mathrm{X}$ & $\mathrm{AO}$ & $\mathrm{CE}, \mathrm{CA}, \mathrm{CH}$ \\
\hline
\end{tabular}


Tabela 3. Continuação...

\begin{tabular}{|c|c|c|c|c|}
\hline & EESGT & $\begin{array}{l}\text { Registros prévios para } \\
\text { a região do Jalapão } \\
\text { (Vitt et al. 2005) }\end{array}$ & $\begin{array}{c}\text { Distribuição } \\
\text { no Cerrado }\end{array}$ & $\begin{array}{c}\text { Distribuição no } \\
\text { continente }\end{array}$ \\
\hline Phalotris labiomaculatus Lema, 2002 & - & $\mathrm{X}$ & $\mathrm{R}$ & $\mathrm{CE}$ \\
\hline Philodryas nattereri Steindachner, 1870 & TO & $\mathrm{X}$ & $\mathrm{AO}$ & $\mathrm{CE}, \mathrm{CA}$ \\
\hline Philodryas patagoniensis Girard, 1858 & - & $\mathrm{X}$ & $\mathrm{AO}$ & $\mathrm{CE}, \mathrm{CA}, \mathrm{MA}, \mathrm{PA}$ \\
\hline Phimophis guerini (Duméril, Bibron \& Duméril, 1854) & - & $\mathrm{X}$ & $\mathrm{AO}$ & $\mathrm{CE}, \mathrm{CH}$ \\
\hline Phimophis iglesiasi (Gomes, 1915) & TO, BA & $\mathrm{X}$ & M & $\mathrm{CE}, \mathrm{CA}$ \\
\hline Pseudoboa nigra (Duméril, Bibron and Duméril, 1854) & & $\mathrm{X}$ & $\mathrm{AO}$ & $\mathrm{CE}, \mathrm{CA}, \mathrm{MA}$ \\
\hline Psomophis joberti (Sauvage, 1884) & - & $\mathrm{X}$ & $\mathrm{AO}$ & $\mathrm{CE}, \mathrm{CA}$ \\
\hline Sibynomorphus mikanii (Schlegel, 1837) & - & $\mathrm{X}$ & $\mathrm{AO}$ & $\mathrm{CE}, \mathrm{CA}, \mathrm{MA}$ \\
\hline Taeniophalus occipitalis (Jan, 1863) & - & $\mathrm{X}$ & $\mathrm{AO}$ & CE, AM \\
\hline Tantilla melanocephala (Linnaeus 1758) & TO, BA & $\mathrm{X}$ & $\mathrm{AO}$ & $\mathrm{CE}, \mathrm{CA}, \mathrm{AM}, \mathrm{MA}$ \\
\hline Thamnodynastes hypoconia (Cope, 1860) & TO & $\mathrm{X}$ & $\mathrm{AO}$ & $\mathrm{CE}, \mathrm{CH}, \mathrm{MA}$ \\
\hline Thamnodynastes cf. pallidus (Linnaeus, 1758) & - & $\mathrm{X}$ & M & $\mathrm{CE}, \mathrm{CA}$ \\
\hline Xenodon merremii (Wagler, 1824) & TO & $\mathrm{X}$ & $\mathrm{AO}$ & $\mathrm{CE}, \mathrm{CA}, \mathrm{CH}, \mathrm{PA}$ \\
\hline \multicolumn{5}{|l|}{ Elapidae } \\
\hline Micrurus brasiliensis Roze, 1967 & TO & $\mathrm{X}$ & $\mathrm{R}$ & $\mathrm{CE}$ \\
\hline \multicolumn{5}{|l|}{ Viperidae } \\
\hline Bothropoides lutzi (Miranda-Ribeiro, 1915) & TO & & $\mathrm{R}$ & $\mathrm{CE}, \mathrm{CA}$ \\
\hline Bothropoides mattogrossensis (Amaral, 1925) & - & $\mathrm{X}$ & $\mathrm{AO}$ & $\mathrm{CE}, \mathrm{CH}$ \\
\hline Bothropoides neuwiedi Wied 1920 & - & $\mathrm{X}$ & $\mathrm{AO}$ & CE \\
\hline Bothropoides pauloensis (Amaral, 1925) & - & $\mathrm{X}$ & $\mathrm{AO}$ & $\mathrm{CE}$ \\
\hline Bothrops moojeni Hoge, 1966 & TO, BA & $\mathrm{X}$ & $\mathrm{AO}$ & $\mathrm{CE}$ \\
\hline Crotalus durissus Linnaeus, 1758 & - & $X$ & $\mathrm{AO}$ & $\mathrm{CE}, \mathrm{CA}, \mathrm{CH}$ \\
\hline \multicolumn{5}{|l|}{ ARCHOSAURIA } \\
\hline \multicolumn{5}{|l|}{ CROCODYLIA } \\
\hline \multicolumn{5}{|l|}{ Alligatoridae } \\
\hline Paleosuchus palpebrosus (Cuvier, 1807) ${ }^{\mathrm{LC}(\mathrm{IUCN})}$ & TO & $\mathrm{X}$ & $\mathrm{AO}$ & $\mathrm{CE}, \mathrm{CA}, \mathrm{MA}, \mathrm{AM}$ \\
\hline Caiman crocodylus (Linnaeus, 1758) & - & $\mathrm{X}$ & $\mathrm{AO}$ & CE, CA, MA, AM \\
\hline \multicolumn{5}{|l|}{ TESTUDINES } \\
\hline \multicolumn{5}{|l|}{ Chelidae } \\
\hline Phrynops geoffroanus (Schweigger, 1812) & - & $\mathrm{X}$ & $\mathrm{AO}$ & $\mathrm{CE}, \mathrm{CA}, \mathrm{MA}$ \\
\hline
\end{tabular}

ampla ocorrência compartilhadas com domínios florestais e abertos adjacentes, representado por 38 espécies $(43,2 \%)$, sendo 9 destas $(10,2 \%)$ compartilhadas exclusivamente com domínios florestais úmidos.

Quando comparado o compartilhamento de espécies de répteis do Jalapão com cada domínio, destaca-se a Caatinga. Das 88 espécies ocorrentes no Jalapão, 40 ocorrem também na Caatinga, 29 na Mata Atlântica, 26 na Amazônia, 22 no Chaco e apenas 5 nos Pampas.

Das espécies registradas para a região, nenhuma está presente na lista de fauna ameaçada de extinção da IUCN. Apenas Paleosuchus palpebrosus foi avaliada (Tabela 3), porém com status de "Least Concern" (LC) ou de menor preocupação (IUCN 2009). A lista das espécies brasileiras de répteis ameaçados do Ministério do Meio Ambiente não inclui nenhuma das espécies da região (Martins \& Molina 2008). Os lagartos dos gêneros Iguana e Tupinambis, e as serpentes da família Boidae estão relacionadas no apêndice II do CITES (Tabela 3), que lista espécies não necessariamente ameaçadas, mas que podem se tornar caso o comércio ou exploração não sejam controlados (CITES 2009).

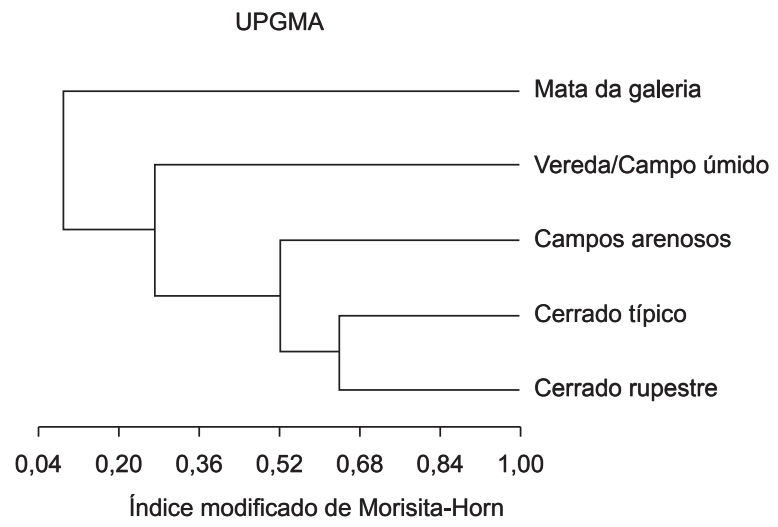

Figura 8. Dendrograma de similaridade faunística resultante de análise de agrupamento entre os habitats amostrados, baseado na riqueza e abundância de Squamata capturados na EESGT.

Figure 8. Dendrogram of faunistic similarity resulted from cluster analysis of the sampled habitats, based on the richness and abundance of the Squamata captured in the EESGT. 
Recoder, R.S. et al.

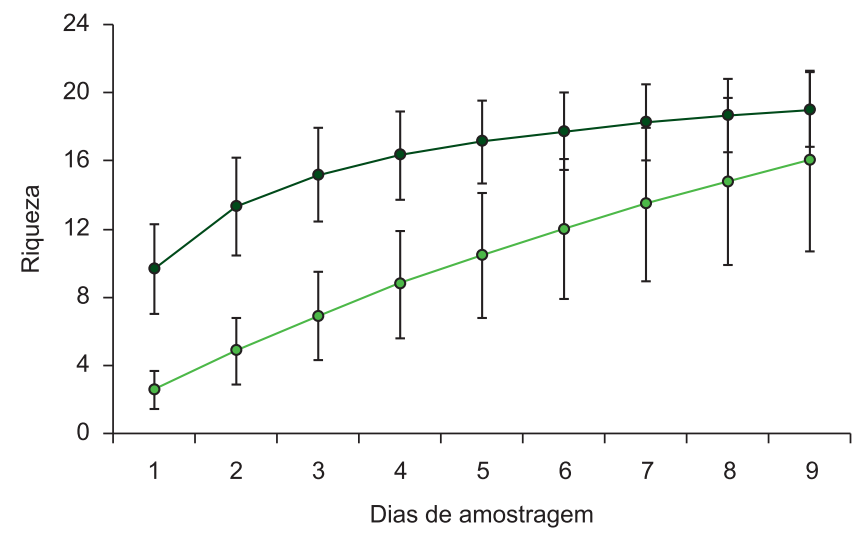

Figura 6. Curva de rarefação com intervalos de confiança para as espécies de lagartos + anfisbenídeos (linha preta) e serpentes (linha verde) amostradas em nove dias de coletas com armadilhas de queda complementadas com busca ativa na porção sul da EESGT (Almas, TO).

Figure 6. Rarefaction curve with confidence intervals for the species of lizards + amphisbaenians (black line) and snakes (green line) from nine days of sampling with pitfall traps supplemented by active search in southern EESGT (Almas, TO).

\section{Discussão}

A riqueza de répteis Squamata registrada para a região da EESGT é alta e comparável à de outras localidades bem amostradas do Cerrado. Levantamentos em regiões de Cerrado com esforços de amostragem semelhantes a este estudo (ao menos 1500 baldes/dia) revelaram níveis de riqueza local de 15 a 28 espécies de lagartos (Pavan \& Dixo 2004, Silva Junior et al. 2005, Vaz-Silva et al. 2007, Nogueira et al. 2009), o que concorda com nossos resultados para cada uma das duas porções da EESGT.

A riqueza observada de serpentes neste estudo representa uma subamostragem do número real estimado de espécies das áreas amostradas, como sugere a ausência de inflexão nas curvas de acumulação local de espécies. Levantamentos com maiores esforços de amostragem em outras localidades do Cerrado apontam riqueza local de 36 a 70 espécies de serpentes (Strüssmann 2000, Pavan \& Dixo 2004, Silva Junior \& Sites Junior 2005, Silva Junior et al. 2005, França \& Araújo 2006, Vaz-Silva et al. 2007, Sawaya et al. 2008, Valdujo et al. 2009). No entanto, considerando os estudos prévios, o registro de 51 espécies de serpentes para o Jalapão caracteriza a região como rica para o grupo.

A amostragem de serpentes é dificultada pela baixa densidade, raridade e modo de vida de muitas espécies e também pela baixa eficiência do tipo de armadilhas como as usadas nesse estudo. Isto explica as curvas de rarefação mais baixas das serpentes em relação aos lagartos e anfisbenas. As armadilhas de interceptação-e-queda, em geral possibilitam que as serpentes de médio e grande porte evitem as quedas ou saiam dos baldes, levando a uma subamostragem do grupo.

A riqueza total de anfisbenídeos registrados é alta, com quatro espécies registradas para a EESGT e um total de seis para a região do Jalapão, uma delas endêmica. Estes valores não destoam de outras localidades do Cerrado (Silva Junior \& Sites Junior 2005, Recoder \& Nogueira 2007, Vaz-Silva et al. 2007), e são comparáveis aos obtidos para a região do Alto Tocantins, GO (Silva Junior et al. 2005) com seis espécies, à região de Palmas, TO com sete espécies (Pavan \& Dixo 2004) e à região do Manso, MT (Strüssmann \& Mott 2009) com oito espécies registradas.

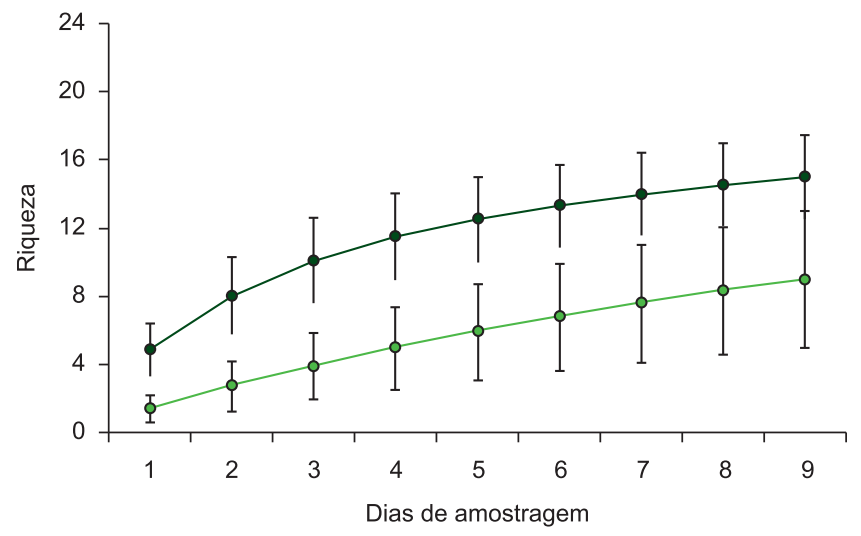

Figura 7. Curva de rarefação com intervalos de confiança para as espécies de lagartos + anfisbenídeos (linha preta) e serpentes (linha verde) amostradas em nove dias de coletas com armadilhas de queda complementadas com busca ativa na porção leste da EESGT (Formosa do Rio Preto, BA).

Figure 7. Rarefaction curve with confidence intervals for the species of lizards + amphisbaenians (black line) and snakes (green line) from nine days of sampling with pitfall traps supplemented by active search in eastern EESGT (Formosa do Rio Preto, BA).

Apesar da alta riqueza de espécies de répteis Squamata registrada na EESGT, os números são ainda subestimados, como pode ser constatado pelas estimativas de riquezas resgatadas que indicam números mais elevados que os observados. Amostragens ulteriores na região vão inevitavelmente acrescentar novos registros de espécies. Esta proposição é sustentada pelo fato de que locais com maiores valores de riqueza de répteis registrados para o Cerrado sejam justamente onde maior esforço foi despendido (e.g. Pavan \& Dixo 2004), geralmente estudos associados a grandes impactos ambientais (Nogueira et al. 2010).

Acrescentamos com este estudo novos registros para a fauna de répteis da EESGT e para a região do Jalapão. Stenocercus quinarius é uma espécie endêmica do Cerrado, conhecida até então da região norte de Minas Gerais e sul da Bahia, habitando tipicamente os "carrascos", cerrados densos e florestas deciduais em planaltos da Serra Geral e adjacências, no contato entre o Cerrado e Caatinga (Nogueira \& Rodrigues 2006, Recoder \& Nogueira 2007, Teixeira Jr. et al. 2008). Tal registro estende em cerca de $350 \mathrm{~km}$ ao norte a distribuição da espécie, sendo importante em vista de sua distribuição relictual no domínio e das poucas localidades de ocorrência conhecidas. O registro na porção leste da EESGT indica que esta espécie está associada às regiões de planalto da Serra Geral, desde sua porção mais austral (próximo à localidade-tipo, na parte sul do Parque Nacional Grande Sertão Veredas) até seu contato com a Chapada das Mangabeiras e depressão do Parnaíba.

Bachia oxyrhina foi descoberta e descrita graças aos esforços de coleta associados a este estudo (ver Rodrigues et al. 2008). A descoberta também revela mais um exemplo de espécie com adaptações específicas a hábitos psamófilos para o Cerrado (Rodrigues et al. 2007, 2008, Ribeiro et al. 2009). Este registro evidencia a falta de conhecimento das faunas associadas aos hábitats arenosos no Cerrado (Rodrigues et al. 2007) frente ao extenso conhecimento da fauna psamófila das Caatingas (Rodrigues 1996). Talvez Bachia oxyrhina seja mais um caso de endemismo para região. Deste modo cinco espécies são conhecidas apenas da região do Jalapão e podem representar casos de endemismos. Essas espécies (Amphisbaena acrobeles, 
Bachia oxyrhina, Cnemidophorus jalapensis, Kentropyx aff. paulensis e Siagonodon acutirostris) destacam a importância histórica e singularidade faunística da região protegida pela estação ecológica.

Cinco espécies de distribuição ampla foram registradas pela primeira vez no Jalapão: Phyllopezus pollicaris, Mabuya frenata, Amphisbaena vermicularis, Atractus pantostictus e Apostolepis ammodites. Registramos também a serpente Apostolepis nelsonjorgei para a região, descrita para Goiás e conhecida de poucas localidades para o Tocantins (Lema \& Renner 2004).

Os padrões de distribuição geográfica das espécies ocorrentes no Jalapão revelam a importância do intercâmbio faunístico com regiões adjacentes para a riqueza regional de espécies. Há predominância de espécies características do Cerrado, porém existe importante contribuição de representantes de domínios adjacentes. Ocorrem principalmente espécies compartilhadas com o domínio das Caatingas. A proximidade geográfica e a dinâmica espacial histórica entre os dois domínios favoreceram a troca faunística, sobretudo na região do planalto dos Gerais onde hábitats de Cerrado e Caatinga se interdigitam (Vanzolini 1976). A contribuição de domínios florestais úmidos é também expressiva. A presença de tributários e cabeceiras de rios pertencentes a duas grandes bacias hidrográficas e as formações florestais associadas favorece a dispersão de fauna florestal para a região. A bacia do Tocantins em especial promove a ocorrência de uma fauna tipicamente amazônica na região do Cerrado (Pavan 2007).

A observação de preferência das espécies por tipos específicos de ambientes, resultando em distribuição não-aleatória na paisagem concorda com estudos prévios realizados em outras áreas de Cerrado (Valdujo 2003, Nogueira et al. 2005, 2009, Pavan 2007, Vitt et al. 2007). A diversidade de tipos de hábitats, justapostos nas paisagens do Cerrado formando um mosaico de ambientes, permite a coexistência local de linhagens com distintas exigências ecológicas, sobretudo no uso de hábitat. A segregação espacial entre as espécies promove assim um incremento na riqueza local de espécies (Colli et al. 2002, Nogueira et al. 2005, 2009).

Ademais da influência dos padrões de segregação ecológica local para a riqueza de répteis na região do Jalapão, padrões de distribuição regional são também notáveis. Na EESGT P. pollicaris foi registrado apenas nas porções leste e central, em considerável abundância, porém estreitamente associada às encostas e afloramentos rochosos adjacentes aos planaltos e morros isolados, como o Morro do Fumo. Surpreendentemente, apesar do grande esforço nesse tipo de ambiente, não registramos $P$. pollicaris na porção sul da estação, onde outra lagartixa, Gymnodactylus amarali, foi abundante.

Cnemidophorus mumbuca e Cnemidophorus jalapensis apesar de utilizarem o mesmo tipo de ambiente, sendo encontrados em campos e cerrados abertos sobre areia, não co-ocorrem. As espécies apresentam distribuição parapátrica (Colli et al. 2009) com C. mumbuca associada ao planalto da Serra Geral e pediplano adjacente e $C$. jalapensis associada a áreas mais baixas na planície de deposição ao oeste do Rio Novo e depressão do Manoel Alves.

Estes exemplos ilustram a importância da heterogeneidade ambiental em escala regional, determinada pela dinâmica espacial e temporal das paisagens, para a riqueza de espécies na região. A diversidade local de répteis é deste modo, condicionada pela interação da segregação ecológica das espécies entre os diferentes tipos de hábitats na paisagem, com a segregação histórica determinada pela heterogeneidade regional, que inclui as mudanças históricas na distribuição dos ambientes e as barreiras e corredores para a dispersão (ver Ricklefs 2008).

Nenhuma das espécies registradas está relacionada como ameaçada em listas de fauna ameaçada de extinção. No entanto, apesar da alarmante taxa de conversão das paisagens do Cerrado, fica evidente que o conhecimento incompleto da diversidade, a escassez de informações robustas sobre a distribuição, dinâmica das populações e requerimentos ecológicos das espécies limita grandemente a avaliação precisa sobre o status de conservação dos répteis do Cerrado.

Em suma, a região do Jalapão possui fauna de répteis rica e característica, que depende da heterogeneidade de hábitats em escala local, da diversidade geomorfológica em escala regional, e do intercâmbio faunístico histórico com regiões adjacentes. A presença de grandes unidades de conservação é, portanto essencial para a preservação da diversidade local e regional de répteis. Também proporciona a oportunidade de estudar aspectos da biologia das espécies em vastas áreas com ambientes naturais ainda pouco perturbados no Cerrado, que podem fornecer subsídios teóricos para medidas adequadas de conservação em áreas impactadas desse ambiente.

Neste contexto a EESGT, por abrigar extensas áreas preservadas de cerrados arenosos e integrar o maior conjunto contínuo de unidades de conservação no domínio, apresentar alta heterogeneidade ambiental e riqueza apreciável de répteis com cinco espécies possivelmente exclusivas da região, possui papel fundamental para a conservação e conhecimento da diversidade de répteis do Cerrado.

\section{Agradecimentos}

Agradecemos ao IBAMA e à Fundação O Boticário de Proteção à Natureza pelo suporte logístico e financiamento durante o levantamento faunístico na EESGT (autorização número 12187-1); também à Conservação Internacional, à Pequi - Pesquisas e Conservação do Cerrado, à Fundação de Amparo à Pesquisa do Estado de São Paulo (FAPESP), e ao Conselho Nacional de Desenvolvimento Científico e Tecnológico (CNPq). Somos gratos a Ana Paula Carmignotto e Luís Fábio Silveira, respectivamente coordenadores dos levantamentos da mastofauna e avifauna, e suas equipes, pela valiosa ajuda em campo. Também aos revisores anônimos que muito contribuíram com correções e sugestões.

\section{Referências Bibliográficas}

AB'SÁBER, A. 1977. Os domínios morfoclimáticos da América do Sul: primeira aproximação. Geomorfologia 52:1-22.

ARRUDA, M.B. \& VON BEHR, M. 2002. Jalapão: Expedição Científica e Conservacionista. IBAMA, Brasília.

CAVALCANTI, R \& JOLY, C.A. 2002. Biodiversity and conservation priorities in the Cerrado region. In The Cerrados of Brazil: Ecology and Natural History of a Neotropical Savanna. (P.S. Oliveira \& R.J. Marquis, eds.). Columbia University Press, New York, p. 351-367.

CITES - Convention on International Trade in Endangered Species of Wild Fauna and Flora. 2009. - Apendix I, II \& III. http://www.cites.org/eng/ app/index.shtml. (último acesso em 26/06/2009).

COLLI, G.R., BASTOS, R.P. \& ARAÚJO, A.F.B. 2002. The character and dynamics of the Cerrado Herpetofauna. In The Cerrados of Brazil: Ecology and Natural History of a Neotropical Savanna. (P.S. Oliveira \& R.J. Marquis, eds.). Columbia University Press, New York, p. 223-241.

COLli, G.R., GIUGLIANO, L.G., MESQUITA, D.O. \& FRANÇA, F.R.G. 2009. A new species of Cnemidophorus from the Jalapão region, in the Central Brazilian Cerrado. Herpetologica 65(3):311-327. doi:10.1655/08049R1.1

COLWELL, R.K. 2006. EstimateS: Statistical estimation of species richness and shared species from samples. Version 8.0. http://purl.oclc.org/ estimates.

EITEN, G. 1972. The cerrado vegetation of Brazil. Bot. Rev. 38(2):201-341. doi:10.1007/BF02859158

FALEIRO, R.P. 2002. Relatório de Técnico-Científico do Levantamento Espeleológico. In Jalapão: Expedição Científica e Conservacionista (M. B. Arruda \& M. von Behr orgs.). IBAMA, Brasília, p.51-81. 
FITCH, H.S. 1949. Road counts of snakes in Western Louisiana. Herpetologica 5(4):87-90.

FRANÇA, F.G.R. \& ARAÚJO, A.F.B. 2006. The conservation status of snakes in Central Brazil. South Am. J. Herpetol. 1(1):25-36.

GOTELLI, N.J. \& COLWELL, R.K. 2001. Quantifying biodiversity: Procedures and pitfalls in the measurement and comparison of species richness. Ecol. Lett. 4:379-391. doi:10.1046/j.1461-0248.2001.00230.x

GOTELLI, N.J. \& ENTSMINGER, G.L. 2008. EcoSim: Null models software for ecology. Version 7. Acquired Intelligence Inc. \& Kesey-Bear. Jericho, VT 05465. http://garyentsminger.com/ecosim.htm.

IUCN - INTERNATIONAL UNION FOR CONSERVATION OF NATURE. 2009. The IUCN Red List of Threatened Species. Versão 2009.1. Disponível em: http://www.iucnredlist.org/ (último acesso em 26/06/2009).

KLINK, C.A. \& MACHADO, R.B. 2005. A conservação do Cerrado brasileiro. Megadiversidade 1(1):147-155.

KLINK, C.A. \& MOREIRA, A.G. 2002. Past and Current Human Occupation, and Land Use. In The Cerrados of Brazil: ecology and natural history of a neotropical savanna (P.S. Oliveira \& R.J. Marquis, eds.). Columbia University Press, New York, p. 69-88.

KOVACH, W.L. 2000. MVSP - A multivariate statistical package for Windows, version 3.1. Kovach Computing Services, Penthraeth.

DE LEMA, T. \& RENNER, M.F. 2004. New species of Apostolepis from Alto Tocantins, Brazil, with comments on the striped pattern species (Serpentes, Elapomorphinae). Biociências 12(2):139-145.

MARES, M.A. 1992. Neotropical mammals and the myth of Amazonian biodiversity. Science 255:976-979. PMid:17793161. doi:10.1126/ science.255.5047.976

MARTINS, M. \& MOLINA, F.B. 2008. Répteis. In Livro Vermelho da Fauna Brasileira Ameaçada de Extinção. Vol. II. (A.B.M. Machado, G.M. Drummond, \& A.P. Paglia, eds.). Ministério do Meio Ambiente, Biodiversidade 19, Brasília, p. 326-376.

MESQUITA, D.O., COLLI, G.R., FRANÇA, F.G.R. \& VITT, L.J. 2006. Ecology of a Cerrado lizard assemblage in the Jalapão Region of Brazil. Copeia (3):460-471. doi:10.1643/0045-8511(2006)2006[460:EOACL A]2.0.CO;2

MITTERMEIER, R.A., GIL, P.R., HOFFMANN, M., PILGRIM, J., BROOKS, T., MITTERMEIER, C.G., LAMOREUX, J. \& DA FONSECA, G.A.B. 2005. Hotspots revisited: Earth's biologically richest and most endangered terrestrial ecoregions. Cemex/Conservation International / Agrupacion, Sierra Madre, Monterrey, Mexico.

MYERS, N., MITTERMEIER, R.A., MITTERMEIER, C.G., DA FONCECA, G.A.B. \& KENT, J. 2000. Biodiversity hotspots for conservation priorities. Nature 403:853-858. PMid:10706275. doi:10.1038/35002501

NIMER, E. 1989. Climatologia do Brasil. IBGE, Departamento de Rescursos Naturais e Estudos Ambientais, Rio de Janeiro.

NOGUEIRA, C. 2006. Diversidade e padrões de distribuição da fauna de lagartos do Cerrado. Tese de doutorado, Instituto de Biociências da Universidade de São Paulo, São Paulo.

NOGUEIRA, C. \& RODRIGUES, M.T. 2006. The genus Stenocercus (Squamata: Tropiduridae) in Extra-Amazonian Brazil, with the description of two new species. South Am. J. Herpetol. 1:149-165. doi:10.2994/18089798(2006)1[149:TGSSTI]2.0.CO;2

NOGUEIRA, C., VALDUJO, P.H. \& FRANÇA, F.G.R. 2005. Habitat variation and lizard diversity in a Cerrado area of Central Brazil. Stud. Neotrop. Fauna Environ. 40:105-112. doi:10.1080/01650520500129901

NOGUEIRA, C., COLLI, G.R. \& MARTINS, M. 2009. Local richness and distribution of the lizard fauna in natural habitat mosaics of the Brazilian Cerrado. Austral Ecology 34:83-96. doi:10.1111/j.14429993.2008.01887.x
NOGUEIRA, C., COLLI, G.R., COSTA, G.C. \& MACHADO, R.B. 2010. Diversidade de répteis Squamata e evolução do conhecimento faunístico no Cerrado In Cerrado: conhecimento científico quantitativo como subsídio para ações de conservação. (I.R. Diniz, J. Marinho-Filho, R.B. Machado \&. R.B. Cavalcanti, eds.). Thesaurus editora, Brasília, p. 333-375.

OLIVEIRA, P.S. \& MARQUIS, R.J. (eds.). 2002. The Cerrados of Brazil: Ecology and Natural History of a Neotropical Savanna. Columbia University Press, New York.

OLIVEIRA-FILHO, A.T. \& RATTER, J.A. 2002. Vegetation physiognomies and woody flora of the Cerrado biome. In The Cerrados of Brazil: Ecology and Natural History of a Neotropical Savanna. (P.S. Oliveira \& R.J. Marquis, eds.). Columbia University Press, New York, p. 91-120.

PAVAN, D. 2007. Assembléias de répteis e anfíbios do Cerrado ao longo do rio Tocantins e o impacto do aproveitamento hidrelétrico da região na sua conservação. Tese de doutorado, Instituto de Biociências da Universidade de São Paulo, São Paulo.

PAVAN, D. \& DIXO, M. 2004. A Herpetofauna da área de influência do reservatório da Usina Hidrelétrica Luís Eduardo Magalhães, Palmas, TO. Humanitas 4/6:13-30.

PROENÇA, C.B., SAMPAIO, A.B., SOARES-E-SILVA, L.H., MILHOMENS, L.C., SIMON, M.F., SIMPSON JUNIOR, P.L. \& FARIAS, R. 2002. Relatório de Botânica. In Jalapão: Expedição Científica e Conservacionista (M. B. Arruda \& von Behr M. orgs.). IBAMA, Brasília, p. 21-28.

RATTER, J.A., RIBEIRO, J.F. \& BRIDGEWATER, S. 1997. The Brazilian Cerrado and threats to its biodiversity. Ann. Bot. 80:223-230. doi:10.1006/ anbo.1997.0469

RECODER, R. \& NOGUEIRA, C. 2007. Composição e diversidade de répteis Squamata na região sul do Parque Nacional Grande Sertão Veredas, Brasil Central. Biota Neotrop. 7(3):267-278. doi: 10.1590/ S1676-06032007000300029.

REDFORD, K.H., TABER, A. \& SIMONETTI, J.A. 1990. There is more to biodiversity than tropical rainforests. Conserv. Biol. 4:328-330. doi:10.1111/j.1523-1739.1990.tb00296.x

RIBEIRO, S.L.B., CASTRO-MELLO, C. \& NOGUEIRA, C. 2009. A new species of Anops Bell, 1833 (Squamata, Amphisbaenia) from Jalapão region in the Brazilian Cerrado. J. Herpetol. 43(1):21-28. doi:10.1670/07299R1.1

RICKLEFS, R.E. 2008. Disintegration of the ecological community. Am. Nat. 172(6):741-50. PMid:18954264. doi:10.1086/593002

RODRIGUES, M.T. 1987. Sistemática, ecologia e zoogeografia dos Tropidurus do grupo torquatus ao sul do rio Amazonas (Sauria, Iguanidae). Arq. Zool. 31(3):105-230.

RODRIGUES, M.T. 1996. Lizards, Snakes and Amphisbaenians from the Quaternary Sand Dunes of the Middle Rio São Francisco, Bahia, Brazil. J. Herpetol. 30(4):513-523. doi:10.2307/1565694

RODRIGUES, M.T. 2005a. Conservação dos répteis brasileiros: os desafios para um país megadiverso. Megadiversidade 1(1):87-94.

RODRIGUES, M.T. 2005b. A biodiversidade dos Cerrados: conhecimento atual e perspectivas, com uma hipótese sobre o papel das matas galerias na troca faunística durante ciclos climáticos. In Cerrado: Ecologia, biodiversidade e conservação. (A. Scariot, J.C.S. Silva \& J.M. Felfili, org.). Ministério do Meio Ambiente, Brasília, p. 235-246.

RODRIGUES, M.T., PAVAN, D. \& CURCIO, F.F. 2007. Two new species of the genus Bachia (Squamata, Gymnophthalmidae) from Central Brazil. J. Herpetol. 41(4):545-553. doi:10.1670/06-103.1

RODRIGUES, M.T., CAMACHO, A., NUNES, P.M.S., RECODER, R.S., TEIXEIRA JUNIOR, M., VALDUJO, P.H., GHELLERE, J.M.B., MOTT, T. \& NOGUEIRA, C. 2008. A new species of the lizard genus Bachia (Squamata: Gymnophthalmidae) from the Cerrados of Central Brazil. Zootaxa 1875:39-50.

SAWAYA, R.J., MARQUES, O.A.V. \& MARTINS, M. 2008. Composition and natural history of a Cerrado snake assemblage at Itirapina, São Paulo state, southeastern Brazil. Biota Neotrop. 8(2):127-149. 
SCARIOT, A., SILVA, J.C.S. \& FELFILI, J.M. (org.). 2005. Cerrado: Ecologia, biodiversidade e conservação. Ministério do Meio Ambiente, Brasília.

SEPLAN -Secretaria do Planejamento e Meio Ambiente do Estado de Tocantins. 2003

SICK, H. 1965. A Fauna do Cerrado. Arq. Zool. 12:71-93.

DA SILVA JUNIOR, N.J. \& SITES JUNIOR, J.W. 2005. Patterns of diversity of neotropical Squamate reptile species with emphasis on the Brazilian Amazon and the conservation potential of indigenous reserves. Conserv. Biol. 9(4):873-901. doi: 10.1046/j.1523-1739.1995.09040873.x

DA SILVA JUNIOR, N.J., SILVA, H.L.R., RODRIGUES, M.T., VALLE, N.C., COSTA, M.C., CASTRO, S.P., LINDER, E.T., JOHANSSON, C. \& SITES JUNIOR, J.W. 2005. A fauna de vertebrados do vale do alto Rio Tocantins em áreas de usinas hidrelétricas. Estudos 32:57-101.

SILVA, J.F., FARIÑAS, M.R., FELFILI, J.M. \& KLINK, C.A. 2006. Spatial heterogeneity, land use and conservation in the cerrado region of Brazil. J. Biogeogr. 33:536-548. doi:10.1111/j.1365-2699.2005.01422.x

STRÜSSMANN, C. 2000. Herpetofauna. In Fauna silvestre da região do Rio Manso, MT. (C.J.R. Alho, coord.). MMA/Edições IBAMA/ ELETRONORTE, Brasília, p.153-189.

STRÜSSMANN, C. \& MOTT, T. 2009. Sympatric amphisbaenids from Manso Dam region, Mato Grosso State, Western Brazil, with the description of a new two-pored species of Amphisbaena (Squamata, Amphisbaenidae). Stud. Neotrop. Fauna E. 44(1):37-46. doi:10.1080/01650520802628295

TEIXEIRA JUNIOR, M., RECODER, R.S. \& RODRIGUES, M.T. 2008. Preliminary data on the natural history of Stenocercus quinarius (Sauria, Tropiduridae) from Peruaçu valley, MG. In Sixth World Congress of Herpetology. 6th World Congress of Herpetology, Manaus.
VALDUJO, P.H. 2003. Distribuição da comunidade de lagartos no mosaico fisionômico do cerrado e suas implicações para a conservação. Dissertação de mestrado, Universidade de Brasília, Brasília.

VALDUJO, P.H., NOGUEIRA, C.C., BAUMGARTEN, L., RODRIGUES, F.H.G., BRANDÃO, R.A., ETEROVIC, A., RAMOS-NETO, M.B. \& MARQUES, O.A.V. 2009. Squamate Reptiles from Parque Nacional das Emas and surroundings, Cerrado of Central Brazil. Checklist 5:405-417.

VANZOLINI, P.E. 1963. Problemas faunísticos do cerrado. In Simpósio sobre o Cerrado. EdUSP, São Paulo, p. 305-321.

VANZOLINI, P.E. 1976. On the lizards of a Cerrado-Caatinga contact: evolutionary and zoogeographical implications (Sauria). Pap. Avulsos Zool. 29:111-119.

VANZOLINI, P.E. 1988. Distributional patterns of South American lizards. In Proceedings of a workshop on Neotropical distributional patterns. (P.E. Vanzolini \& W.R. Heyer, eds.). Academia Brasileira de Ciências, Rio de Janeiro, p. 317-342.

VAZ-SILVA, W., GUEDES, A.G., AZEVEDO-SILVA, P.L., GONTIJO, F.F., BARBOSA, R.S., ALOÍSIO, G.R. \& OLIVEIRA, F.C.G. 2007. Herpetofauna, Espora Hydroelectric Power Plant, state of Goiás, Brazil. Check List 3(4):338-345.

VITT, L.J. 1991. An introduction to the ecology of the Cerrado lizards. J. Herpetol. 25:79-90. doi:10.2307/1564798

VITT, L.J., CALDWELL, J.P., COLLI, G.R., GARDA, A.A., MESQUITA, D.O., FRANÇA, F.G.R., SHEPARD, D.B., COSTA, G.C., VASCONCELLOS, M.M. \& DE NOVAES E SILVA, V. 2005. Uma atualização do guia fotográfico de répteis e anfíbios da região do Jalapão no Cerrado Brasileiro. Occas. Pap. / Sam Noble Oklahoma Mus. Nat. Hist. 2:1-24.

VITT, L.J., COLLI, G.R., CALDWELL, J.P., MESQUITA, D.O., GARDA, A.A. \& FRANÇA, F.G.R. 2007. Detecting variation in microhabitat use in low diversity lizard assemblages across small-scale habitat gradients. J. Herpetol. 41:653-662. doi: 10.1670/06-279.1

Recebido em 08/04/2010 Versão reformulada recebida em 11/03/2011 Publicado em 18/03/2011 